\title{
Chemical evolution in Sérsic 159-03 observed with XMM-Newton
}

\author{
J. de Plaa ${ }^{1,2}$, N. Werner ${ }^{1}$, A. M. Bykov ${ }^{3}$, J. S. Kaastra ${ }^{1}$, M. Méndez ${ }^{1}$, J. Vink ${ }^{1,2}$, J. A. M. Bleeker ${ }^{1,2}$, \\ M. Bonamente ${ }^{4}$, and J. R. Peterson ${ }^{5}$ \\ 1 SRON Netherlands Institute for Space Research, Sorbonnelaan 2, 3584 CA Utrecht, The Netherlands \\ e-mail: j.s.kaastra@sron.nl \\ 2 Astronomical Institute, Utrecht University, PO Box 80000, 3508 TA Utrecht, The Netherlands \\ 3 A.F. Ioffe Institute for Physics and Technology, 26 Polytechnicheskaia, 194021 St. Petersburg, Russia \\ 4 Department of Physics, University of Alabama, Huntsville, AL 35899, USA \\ 5 KIPAC, Stanford University, PO Box 90450, MS 29, Stanford, CA 94039,USA
}

Received 20 July 2005 / Accepted 26 February 2006

\section{ABSTRACT}

\begin{abstract}
Using a new long X-ray observation of the cluster of galaxies Sérsic 159-03 with XMM-Newton, we derive radial temperature and abundance profiles using single- and multi-temperature models. The fits to the EPIC and RGS spectra prefer multi-temperature models especially in the core. The radial profiles of oxygen and iron measured with EPIC/RGS and the line profiles in RGS suggest that there is a dip in the $\mathrm{O} / \mathrm{Fe}$ ratio in the centre of the cluster compared to its immediate surroundings. A possible explanation for the large scale metallicity distribution is that SNIa and SNII products are released in the ICM through ram-pressure stripping of in-falling galaxies. This causes a peaked metallicity distribution. In addition, SNIa in the central cD galaxy enrich mainly the centre of the cluster with iron. This excess of SNIa products is consistent with the low $\mathrm{O} / \mathrm{Fe}$ ratio we detect in the centre of the cluster. We fit the abundances we obtain with yields from SNIa, SNII and Population-III stars to derive the clusters chemical evolution. We find that the measured abundance pattern does not require a Population-III star contribution. The relative contribution of the number of SNIa with respect to the total number of SNe which enrich the ICM is about $25-50 \%$. Furthermore, we discuss the possible presence of a non-thermal component in the EPIC spectra. A potential source of this non-thermal emission can be inverse-Compton scattering between Cosmic Microwave Background (CMB) photons and relativistic electrons, which are accelerated in bow shocks associated with ram-pressure stripping of in-falling galaxies.
\end{abstract}

Key words. galaxies: clusters: general - galaxies: clusters: individual: Sérsic 159-03 - galaxies: abundances intergalactic medium - X-rays: galaxies: clusters

\section{Introduction}

Hot diffuse X-ray emitting gas dominates the visible mass in clusters of galaxies, but the structure and evolution of the cluster is not yet fully understood. During its formation, supernova explosions and galactic winds of member galaxies have enriched the Intra-Cluster Medium (ICM) substantially (De Young 1978). The abundances can provide the relative contribution of Supernova type Ia (SNIa), Supernova type II (SNII) and population-III stars (PopIII) to the enrichment of the ICM (e.g. Iwamoto et al. 1999; Tsujimoto et al. 1995), because the abundance ratios of the various elements are signatures of supernova types Ia and II and possibly of the remains of PopIII stars (e.g. Gibson et al. 1997; Loewenstein 2001; Baumgartner et al. 2005). The radial distribution of the metals provides information about dynamical ways to enrich the ICM like, for example, ram-pressure stripping (e.g. Schindler et al. 2005).

Clusters of galaxies appear as knots in the cosmic web. They accrete gas from the surrounding filaments, which consist of warm gas with a temperature in the range of $10^{5-6} \mathrm{~K}$. According to numerical hydro-dynamical simulations by e.g. Cen \& Ostriker (1999) and Davé et al. (2001), this Warm-Hot Intergalactic Medium (WHIM) could contain about half of the missing baryons in the universe,

There have been several attempts to detect this WHIM in emission. In the late 90's Lieu et al. (1996) and Mittaz et al. (1998) discovered a soft X-ray excess in EUV and
ROSAT spectra of several clusters. More recent observations with XMM-Newton appear to confirm the presence of a soft excess in some cluster spectra (Kaastra et al. 2003; Finoguenov et al. 2003). The detection of a possibly redshifted O VII line, which traces gas with a temperature of $\sim 10^{6} \mathrm{~K}$, suggests that the gas might be the WHIM. Unfortunately, current instruments do not have sufficient spectral resolution to prove that the emission is indeed extragalactic.

Recent measurements of Sérsic 159-03 by Bonamente et al. (2005) and Kaastra et al. (2003) show that the soft-excess can be fit both using thermal and non-thermal models. InverseCompton scattering of CMB photons with relativistic electrons can also contribute a non-thermal power-law component to the spectra. This mechanism was already proposed by Sarazin \& Lieu (1998) to explain the extreme-ultraviolet emission from clusters. In the hard X-ray band up to $80 \mathrm{keV}$, detections of nonthermal emission have been claimed in several clusters, for example Coma and Abell 2256 (Fusco-Femiano et al. 1999, 2005). But these BeppoSAX detections of the hard-excess are still subject to debate (Rossetti \& Molendi 2004).

The cluster of galaxies Sérsic 159-03, also known as ACO S 1101, was discovered by Sérsic (1974). Since then it was studied in X-rays as part of several cluster samples: e.g. EXOSAT (Edge \& Stewart 1991) and ROSAT (Allen \& Fabian 1997). Kaastra et al. (2001) reported results from an XMM-Newton observation with a useful exposure time of about $35 \mathrm{ks}$ showing a radial temperature profile which peaks 
at $k T=2.7 \mathrm{keV}$ at a radius of $2^{\prime}$ from the core. The temperature drop in the core is relatively modest, while the temperature outside the $2^{\prime}$ radius drops rapidly to values around $0.5 \mathrm{keV}$. Because Sérsic 159-03 is thought to show a large soft X-ray excess, the cluster was also included in the sample of Kaastra et al. (2003).

In this paper we present results from a $121 \mathrm{ks}$ long XMMNewton (Jansen et al. 2001) observation of Sérsic 159-03. The main goal of the paper is to obtain accurate temperature and abundance profiles as far out from the core as possible using a new method for handling the background. We exploit the large effective area of XMM to obtain temperature and abundance profiles with EPIC (Turner et al. 2001) and RGS (den Herder et al. 2001). This deep observation also allows us to study the nature of the previously detected soft excess in more detail, and in addition it provides more accurate radial profiles of the temperature and metal abundances. We then fit the derived abundances to yields of Supernovae type Ia and II, and PopIII stars. Moreover, we discuss the potential presence of non-thermal emission in the cluster.

Throughout this paper we use $H_{0}=70 \mathrm{~km} \mathrm{~s}^{-1} \mathrm{Mpc}^{-1}, \Omega_{\mathrm{m}}=$ 0.3 and $\Omega_{\Lambda}=0.7$. Using this cosmology $1^{\prime}$ is $73 \mathrm{kpc}$ at the cluster redshift of 0.0564 (Maia et al. 1987). The elemental abundances presented in this paper are given relative to the solar abundances from Lodders (2003).

\section{Observations and data analysis}

The XMM-Newton observation of Sérsic 159-03 was performed on November 20, 2002 and had a total duration of $121 \mathrm{ks}$. The two EPIC MOS cameras were operated in Full Frame mode and the EPIC pn camera in Extended Full Frame mode. For all EPIC cameras the thin filter was used. The RGS instruments were operated in the standard spectroscopy mode. All data were analysed with the 6.1.0 version of the XMM Science Analysis System (SAS).

\subsection{EPIC analysis}

One of the most important things to account for in extended source analysis is the background. Because we intend to measure the cluster properties as far from the core of the cluster as possible, we need an accurate estimate of the local background, especially for the dim outer parts of the cluster. In general the source fills the entire field of view, which makes a direct measurement of the local background very difficult. Common practice is to extract spectra from a combined event list of several observations of empty fields, like the ones compiled by Lumb et al. (2002) or Read \& Ponman (2003), and use them as best estimate for the local background. The spectra extracted from this blank field are scaled and then subtracted from the source spectra. This method works fine in areas in which the surface brightness of the source is high, and with clusters that have similar background conditions (e.g. particle background, local cosmic background and instrumental background) compared to the blank fields.

The Cosmic X-ray Background (CXB), however, consists of multiple components, which are more extensively described in Sect. 2.1.3. The emission can be roughly divided in two parts: a soft thermal component originating from hot plasma in our own galaxy and a non-thermal power-law component caused by unresolved distant point sources, predominantly AGN. The soft galactic CXB component varies spatially across the sky.

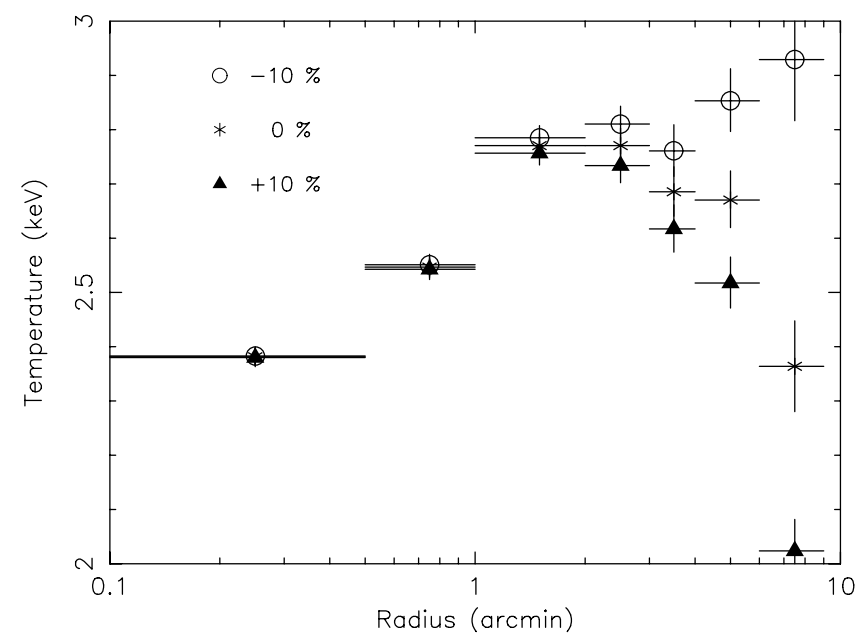

Fig. 1. Systematic effects in a temperature profile obtained with a blanksky background dataset (Read \& Ponman 2003). The points with the cross are derived from spectra with no background scaling. The background for the circled data points is scaled with 0.9 and the triangles with 1.1 .

Therefore, the total photon background at low energies can vary up to $30-60 \%$ from pointing to pointing (Read \& Ponman 2003). It is clear that when the background conditions in the source observation are very different from the average blank-sky background, this can lead to systematic uncertainties in the fitted parameters from the outer parts of the cluster.

In Fig. 1 we show the systematic effect of background subtraction on a temperature profile extracted from the EPIC data of Sérsic 159-03. For this plot we deliberately introduced an error of $+10 \%$ and $-10 \%$ in the background normalisation. The crosses show a profile derived with no background scaling at all, while the circles and triangles show the temperature profile for a scaling of $-10 \%$ and $+10 \%$, respectively. From this plot we see that overestimating the background results in a lower temperature, and vice versa. Note that we included a $10 \%$ systematic error on the background during fitting. We conclude that the uncertainties in the temperature are mainly caused by the background scaling and therefore we can gain a lot in accuracy by constraining the background normalisations.

In order to find the best estimate for the local background we divide the background into three components: soft-protons, instrumental background, and the CXB. By separating these components in our analysis, we will be able to constrain the normalisation and accuracy of each component. The method we use is similar to the one used by De Luca \& Molendi (2004), but with a few modifications. The steps are described below.

\subsubsection{Soft protons}

In order to minimise the effect of soft protons in the spectra, we cut out the time intervals which show enhanced soft-proton flux using the method described in Pratt \& Arnaud (2002). We make a light curve using the events with energies between $2-12 \mathrm{keV}$ and with a bin size of $100 \mathrm{~s}$. From this light curve we extract the distribution of counts per bin. The resulting histogram is then fitted with a Poissonian function to determine the average number of counts per bin. The thresholds are subsequently fixed to $N \pm 2 \sqrt{N}$, where $N$ is equal to the mean number of counts within a $100 \mathrm{~s}$ bin. This way we cut out every flare 
Table 1. The closed filter observations that we use for the EPIC background subtraction.

\begin{tabular}{llll}
\hline \hline Instrument & Obs. date & Obs. ID & Exposure \\
\hline MOS & $2003-04-06$ & 0150390101 & $200 \mathrm{ks}$ \\
pn & $2003-08-12$ & 0160362801 & $35 \mathrm{ks}$ \\
\hline
\end{tabular}

which reaches the $2 \sigma$ level. This method is more strict in rejecting soft-proton flares than using the conventional $>10 \mathrm{keV}$ light curve with $3 \sigma$ clipping. Because of the broad energy interval we choose, the signal-to-noise in the light curve is very high and even small flares stand out from the quiescent level. By putting a strict $2 \sigma$ threshold we exclude also small flares without losing a lot of usable data ( $4 \%$ max. is lost in this case). This way we minimise the effect of soft-proton flares which can bias our temperature determination like in Fig. 1. After applying the threshold we obtain an effective exposure time of $81 \mathrm{ks}$ for both MOS instruments and $60 \mathrm{ks}$ for $\mathrm{pn}$.

The disadvantage of this method is that we do not necessarily subtract the quiescent level of soft protons. We can get an indication of the magnitude of the soft-proton count rate by using CCD9 of the RGS instrument. For this CCD the photon count rate from the source is very low, so the count rate is dominated by soft protons and instrumental noise. For our observation we find that the RGS CCD9 count rate is $0.086 \mathrm{cnts} / \mathrm{s}$ in the quiescent periods. Compared to all the soft-proton background count rates measured during the lifetime of XMM-Newton, this residual soft proton background is low (González-Riestra 2004). Therefore, the influence on our analysis should be very small.

\subsubsection{Instrumental background}

A good template for the instrumental background can be obtained from a closed-filter observation. For this analysis we use a closed-filter observation which was performed after the cooling of the EPIC and RGS instruments in November 2002. The properties of the observations are listed in Table 1.

The instrumental background contains roughly two components: fluorescence lines and a power law. A study of the EPIC-pn background by Katayama et al. (2004) shows that the variation of the fluorescence lines is much lower than the variation in the continuum. We use the power-law component to model the variable hard-particle background and the intrinsic instrumental noise. This background component is mainly caused by hard-particles which are able to reach the detector even when the filter wheel is in closed position. We especially choose a long closed-filter observation, not taken during the passage of the Earth radiation belts, to avoid getting a too large variation in the hard-particle flux with respect to our Sérsic 159-03 observation.

Because these variations can have a significant effect on fitted parameters (see Fig. 1), we would like to be able to modify the normalisation of the hard-particle power law by a few percent without changing the normalisations of the instrumental lines. In order to find the scaling factors for this normalisation, we can use the events registered outside the field of view (out-of-FOV) of the EPIC instruments in both the closed-filter and source observations. These non-illuminated parts of the CCD chips provide unbiased data on the power-law normalisation, also when a bright source is present in the field of view.

We start by determining the power-law index of the instrumental power law by fitting a closed-filter spectrum extracted from the full field of view. We assume that this power-law index will be constant over the whole detector, since the observation does not suffer from vignetting and PSF effects of the mirror. We fit the spectrum with just a spectral redistribution file and no effective area file. From these fits we obtain a photon index $(\Gamma)$ of 0.15 for MOS and 0.37 for pn. The MOS value is consistent with the value of $\sim 0.2$ obtained in the same way by De Luca \& Molendi (2004)

We extract the events labelled as out-of-FOV events (FLAG==\#XMMEA_16) to determine the normalisation of the instrumental background. To be sure that photons and soft-protons that scatter into the shielded out-of-FOV region of the detector are not polluting the measurement, we only count events registered outside a radius of $15.4^{\prime}$ from the centre of the field-ofview. We divide the out-of-FOV count rates in our Sérsic 159-03 observation with the closed-filter out-of-FOV count rates in the 8-12 keV band to obtain an instrumental background scaling factor $(c)$. The $c$ values are $1.03 \pm 0.02,0.97 \pm 0.02$ and $0.97 \pm 0.06$ for MOS1, MOS2 and pn respectively. In this case the correction on the power-law normalisation is only $\pm 3 \%$ and within the statistical error, but we prefer to use the most likely value for $c$. Despite the fact that our values for $c$ are consistent with being 1 , this is a useful exercise, because the normalisation of closed filter spectra is known to vary by about $15 \%$ (De Luca \& Molendi 2004). Already a deviation of a few percent can lead to discrepancies in the temperature determination.

We cannot simply multiply the closed-filter spectra with our values for $c$, because then the instrumental lines would not be well subtracted. The only component that we have to scale is the power-law component. Therefore, we add (or subtract) a power law with the same slope $(\Gamma)$ to the original closed filter spectra $\left(S_{\text {orig }}\right)$. The normalisation of the power law $(n)$ we add is derived as follows. We first solve this simple system for $S_{\text {orig }}$ to approximate the scaled spectrum $S_{\text {scal }}$ :

$$
\left\{\begin{array}{l}
S_{\text {scal }}=c S_{\text {orig }} \\
S_{\text {scal }}=n E^{-\Gamma}+S_{\text {orig }}
\end{array}\right.
$$

and work out $n$ :

$n=\frac{(c-1)}{E^{-\Gamma}} S_{\text {orig }}$.

We can subsequently calculate $n$ for every energy bin of energy $E$. We exclude energy intervals affected by instrumental lines. The mean value of $n$ over all these bins is determined by fitting a Gaussian to a histogram of $n$. This value will be used to add or subtract the power law from the closed filter spectra. Finally, we subtract this scaled spectrum from the source spectra.

\subsubsection{Cosmic X-ray background}

Instead of subtracting the CXB from the spectra, like it is done with blank-field data, we include the $\mathrm{CXB}$ components during fitting. Because the CXB can vary spatially across the sky we can more easily adapt the flux of each component to the local conditions. Unlike the case of the instrumental background and soft-protons, all the photons enter through the XMM mirrors in the same way as the source photons. Therefore, the response files (rmf) and effective area files (arf) must be applied and we must fit the CXB simultaneously with the source spectra.

For the fitting we use the spectral components described by Kuntz \& Snowden (2000) and De Luca \& Molendi (2004). For the thermal components in this paper we use MEKAL models, but the thermal components in Kuntz \& Snowden (2000) are fitted with Raymond-Smith models (Raymond \& Smith 1977). 
Table 2. The CXB background components we use in the fits of Sérsic 159-03. The integrated absorbed intensity over the $0.3-10 \mathrm{keV}$ energy range was calculated using an $N_{\mathrm{H}}$ value of $1.79 \times 10^{20} \mathrm{~cm}^{-2}$. In the thermal components the abundances are set to solar values.

\begin{tabular}{lllll}
\hline \hline Component & $\begin{array}{l}\mathrm{kT} \\
(\mathrm{keV})\end{array}$ & $\Gamma$ & $\mathrm{Z}_{\odot}$ & $\begin{array}{l}\text { Integrated intensity } \\
\left(\mathrm{erg} \mathrm{cm}^{-2} \mathrm{~s}^{-1} \mathrm{deg}^{-2}\right)\end{array}$ \\
\hline Soft thermal & 0.070 & & 0.3 & $2.23 \times 10^{-12}$ \\
Hard thermal & 0.20 & & 1.0 & $1.03 \times 10^{-11}$ \\
Power law & & 1.41 & & $3.16 \times 10^{-11}$ \\
\hline
\end{tabular}

There are, however, significant differences in ionisation balance and line strengths between MEKAL and Raymond-Smith, because the MEKAL code includes many more ions and up-todate atomic constants. Hence, a temperature determined from a spectrum using the Raymond-Smith model is different from a temperature determined using MEKAL for the same dataset. To obtain consistency, we choose to convert the model temperatures from Kuntz \& Snowden (2000) to MEKAL temperatures to match the measured spectra by Kuntz \& Snowden (2000) in our modelling. Unfortunately, this transformed Kuntz \& Snowden (2000) model does not fit a spectrum extracted from the Read \& Ponman (2003) blank-fields. Therefore, we empirically fit the Read \& Ponman (2003) blank-fields with a power law, a soft, and a hard thermal component, which turns out to be a good description of the data. Based on the best fit and the model by Kuntz \& Snowden (2000) we fix the temperatures and metallicities to the values listed in Table 2.

In order to estimate the normalisations of the local background components around Sérsic 159-03 we fit them to the outermost annulus (9-12' from the core of the cluster) using the temperatures derived from the Read \& Ponman (2003) blankfields. We add an additional thermal component for the cluster emission. In our final fits for all annuli we fix the normalisations of the background components to the fitted values of the 9-12' annulus, which are listed in Table 2. For comparison, the 2-10 keV absorbed integrated intensity of the power-law component is $2.26 \times 10^{-11} \mathrm{erg} \mathrm{cm}^{-2} \mathrm{~s}^{-1} \mathrm{deg}^{-2}$, which is consistent with the value of $(2.24 \pm 0.16) \times 10^{-11} \mathrm{erg} \mathrm{cm}^{-2} \mathrm{~s}^{-1} \mathrm{deg}^{-2}$ found by De Luca \& Molendi (2004).

In order to avoid large background fluctuations in our extraction regions we cut out bright point sources which were identified by eye. The unresolved point sources are taken into account by the CXB power law.

\subsubsection{Data extraction and fitting}

As we are interested in the spectral parameters as a function of radius, we extract spectra from annuli as defined in Table 3 . To gain statistics we choose a width for the annuli which is $\geq 30^{\prime \prime}$. By using these relatively wide annuli, we are also less sensitive to the energy dependent shape of the PSF and therefore we neglect this effect in the rest of the analysis. Because of small calibration differences between MOS and pn we include a 5\% systematic error on the source and background spectra. This error is added in quadrature to the poissonian error of the data points.

\subsection{RGS analysis}

We extract the RGS spectra following the method described in Tamura et al. (2001). In addition, we do spatially resolved spectroscopy using the RGS data: with RGS it is possible to extract spectra from different regions in the inner $4^{\prime}$ of the cluster. The
Table 3. Boundaries of the annular extraction regions used in our EPIC analysis. The annuli are centred on the cluster centre.

\begin{tabular}{ccc}
\hline \hline Annulus & Inner boundary $\left(^{\prime}\right)$ & Outer boundary $\left(^{\prime}\right)$ \\
\hline 1 & 0.0 & 0.5 \\
2 & 0.5 & 1.0 \\
3 & 1.0 & 2.0 \\
4 & 2.0 & 3.0 \\
5 & 3.0 & 4.0 \\
6 & 4.0 & 6.0 \\
7 & 6.0 & 9.0 \\
\hline
\end{tabular}

different regions can only be separated if they lie along the crossdispersion axis of the instrument. We select the events from several rectangular areas on the CCD strip in the cross-dispersion direction. In Fig. 2 we show how these extraction regions are projected on the sky (MOS1, upper panel) and on the chips of the RGS (lower panel).

Because the cluster fills the entire field-of-view of the RGS, we need a blank field observation to extract the background spectrum. For this observation, which was taken prior to the cooling of RGS1 but after cooling of RGS2, we choose a Lockman Hole observation with an effective exposure time of $100 \mathrm{ks}$ performed just 3 XMM-Newton orbits after our observation, also still before the cooling of RGS1. This way we minimise systematic effects due to hot pixels and instrument response. The flare subtraction is analogous to the method used with EPIC, but now we use the events from CCD 9 outside the central area. We select only events that have a position greater than $30^{\prime \prime}$ from the dispersion axis to make the light curve. This method was applied to both source and background datasets.

The RGS spectrometer operates without a slit. This means that all photons from within the (in our case) $5^{\prime} \times \sim 12^{\prime}$ field of view end up in the final spectrum, but not necessarily at the right wavelength. Only photons which are emitted in the cluster centre end up at the dispersion coordinate which corresponds to the correct wavelength. If the photon originates from the outskirts at an angle $\theta$ (projected on the dispersion axis) from the cluster centre, then the instrument will register it at a different dispersion coordinate and assign a wavelength to it which is shifted with respect to the true wavelength. This shift in wavelength depends linearly on incidence angle $\theta$ (arcmin) projected on the dispersion axis:

$\Delta \lambda=0.138 \AA \Delta \theta$.

Because of this effect, the line-emission appears to be broadened depending on the spatial extent of the source along the dispersion direction (see Davis 2001, for a complete discussion about grating responses).

In order to describe the data properly, the spectral fits need to account for the effect described above. In practice, this is accomplished by convolving the spectral models with the surface brightness profile of the source along the dispersion direction (Tamura et al. 2004). Therefore, we extract the cluster intensity profile from MOS1 along the dispersion direction of RGS. For each extraction region we can convert the spatial profile, which is a function of $\theta$, into a line profile in $\AA$ using Eq. (3). We convolve this profile with the model spectrum during spectral fitting. However, the width of the measured line profile can be different from the line profile that we derive from MOS1, because the surface brightness at the wavelength of a spectral line is not necessarily the same as the surface brightness in a broad energy band. To take this effect into account, we multiply the wavelength axis 

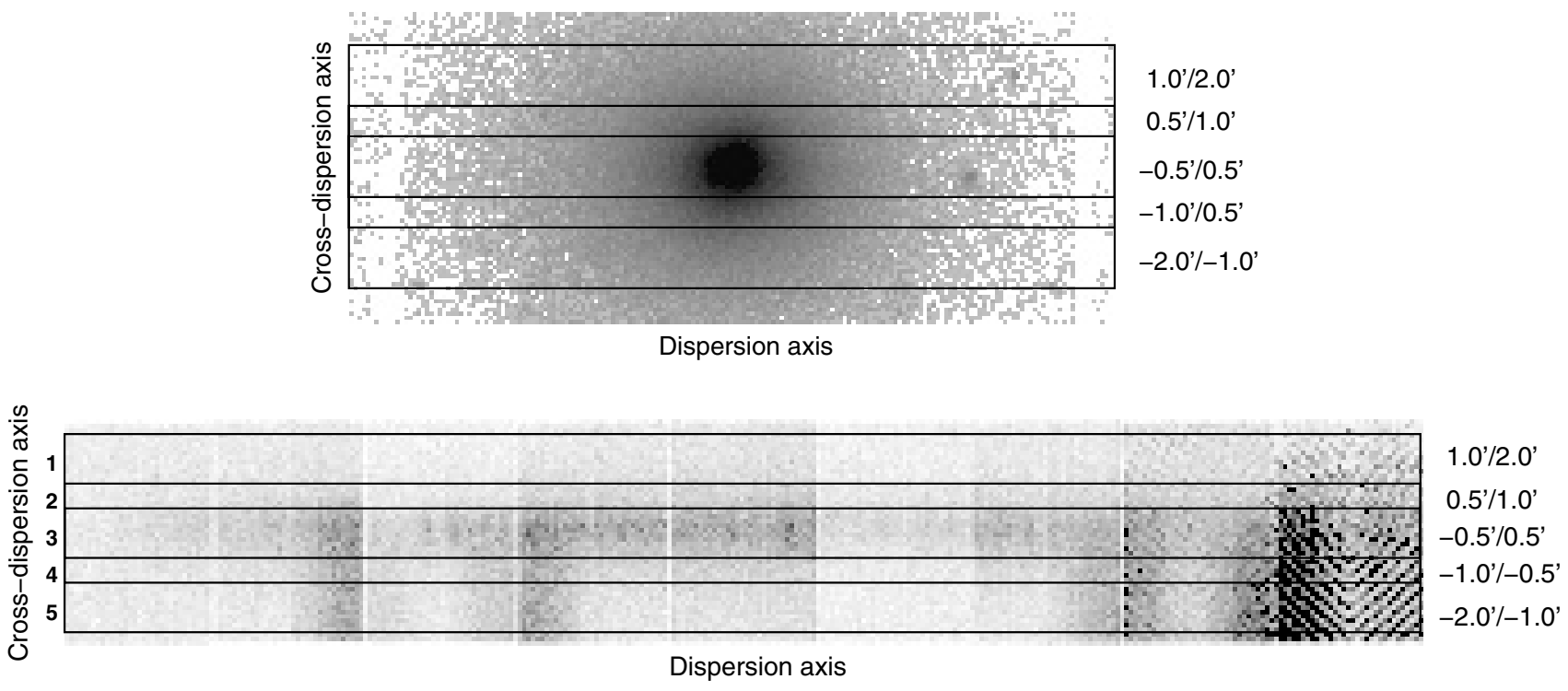

Fig. 2. RGS extraction regions projected on top of a MOS1 image (top panel) and projected on top of a combined RGS1/RGS2 detector image (bottom panel). In the eighth and ninth CCD of RGS the instrumental effect of fixed-pattern noise causes the fish-bone shaped patterns.

of the derived line profile with a scale factor. A scale factor of 1.0 corresponds directly to the wavelength scale of the profile we derive from the continuum. A scale factor of 2.0 stretches the line profile in wavelength space by a factor of two compared to the original line profile. In this way, we are able to fit the widths of the line profiles and to have a measure of the spatial extent of the line-emission region.

\section{Spectral models}

In our analysis we fit several models to the spectra using the SPEX package (Kaastra et al. 1996). These models can be a combination of a number of thermal models (MEKAL) and a power-law model. Two models, however, include a more sophisticated combination of thermal models that we call Differential Emission Measure (DEM) models. Previous papers (e.g. Peterson et al. 2003; Kaastra et al. 2004; de Plaa et al. 2004) show that many clusters can be fitted better when we use a distribution of temperatures instead of a single temperature model. Actually, we expect that the plasma within our annuli should contain multiple temperatures and not one. An observational proof of this is given in Werner et al. (2006). In the case of Sérsic 159-03 we fit two types of distributions: a truncated power-law distribution (wdem) and a Gaussian distribution $($ gdem).

\subsection{WDEM}

We use the so-called wdem model, where the emission measure, $Y=\int n_{\mathrm{e}} n_{\mathrm{H}} \mathrm{d} V$, of a number of thermal components is distributed as a truncated power law. This is shown in Eq. (4) adapted from Kaastra et al. (2004):

$$
\frac{\mathrm{d} Y}{\mathrm{~d} T}= \begin{cases}c T^{1 / \alpha} & \beta T_{\max } \leq T<T_{\max } \\ 0 & T>T_{\max } \vee T<\beta T_{\max } .\end{cases}
$$

This distribution is cut off at a fraction of $T_{\max }$ which is $\beta T_{\max }$. The value of $\beta$ is set to 0.1 in this study. The model above is an empirical parametrisation of the DEM distribution found in the core of many clusters. In this form the limit $\alpha \rightarrow 0$ yields the isothermal model at $T_{\max }$.
In order to compare the outcome of the wdem model with single-temperature models, we can calculate the emission weighted mean of the DEM distribution. The mean temperature $k T_{\text {mean }}$ follows from Eq. (5):

$T_{\text {mean }}=\frac{\int T \frac{\mathrm{d} Y}{\mathrm{~d} T} \mathrm{~d} T}{\int \frac{\mathrm{d} Y}{\mathrm{~d} T} \mathrm{~d} T}$.

When we integrate this equation between $\beta T_{\max }$ and $T_{\max }$, we obtain a direct relation between $T_{\text {mean }}$ and $T_{\max }$ as a function of $\alpha$ and $\beta$ :

$T_{\text {mean }}=\frac{(1+1 / \alpha)}{(2+1 / \alpha)} \frac{\left(1-\beta^{1 / \alpha+2}\right)}{\left(1-\beta^{1 / \alpha+1}\right)} T_{\max }$.

The values for $k T_{\text {mean }}$ we present in this paper are calculated using Eq. (6).

A detailed comparison of the wdem model with the classical cooling-flow model can be found in de Plaa et al. (2005). In general the wdem model contains less cool gas than the classical cooling-flow model, which is consistent with recent observations (Peterson et al. 2001, 2003).

\subsection{GDEM}

Another DEM model that we use is a Gaussian differential emission measure distribution, gdem, in $\log T$ :

$Y(x)=\frac{Y_{0}}{\sigma_{\mathrm{T}} \sqrt{2 \pi}} \mathrm{e}^{-\left(x-x_{0}\right)^{2} / 2 \sigma_{\mathrm{T}}^{2}}$.

In this equation $x=\log T$ and $x_{0}=\log T_{0}$ where $T_{0}$ is the average temperature of the distribution. The width of the Gaussian is $\sigma_{\mathrm{T}}$. Compared to the wdem model this distribution contains more emission measure at higher temperatures.

\section{Results}

\subsection{EPIC/RGS spectral fitting}

In this section we fit both the EPIC and RGS spectra with the models described in Sect. 3. From the fits we obtain temperatures 
and abundances for which we also derive spatial information. In all the fits we fix $N_{\mathrm{H}}$ to $1.79 \times 10^{20} \mathrm{~cm}^{-2}$ which is the value deduced from H I data (Dickey \& Lockman 1990). This value is the same as the one used in Kaastra et al. (2004). We use the Verner et al. (1996) cross-sections in our absorption model. Throughout the paper we use errors at the $\Delta \chi^{2}=1(68 \%)$ level for one interesting parameter.

The EPIC spectra are rebinned to the optimal binning of $\sim 1 / 3 F W H M$ of the resolution of the instrument. We use the response matrix to derive the $F W H M$ for every energy. The spectra are fitted over the $0.3-10 \mathrm{keV}$ range. We are aware that below $0.6 \mathrm{keV}$ there are some calibration uncertainties of the order of $5-10 \%$ when using SAS 6.1.0. This is partially accounted for by the $5 \%$ systematic error that we add to the spectra during fitting. The remaining calibration uncertainties are still small compared to the uncertainties in the soft X-ray background. Because of the importance of the soft-excess problem and the role of O VII we choose to include the $0.3-0.6 \mathrm{keV}$ energy band in our fits.

For RGS we discuss the spectrum which was extracted from a $4^{\prime}$ wide strip in the cross-dispersion direction, with which we obtain the highest signal-to-noise. In addition we also present spatially-resolved spectra extracted from smaller strips in the cross-dispersion direction. This provides a radial profile of the temperature and abundances in the core region $\left(0-2^{\prime}\right)$.

After preliminary analysis, the RGS spectra show a discrepancy with any thermal model around 29-33 $\AA$. The data points are significantly below the models in this interval, as can be seen in Fig. 5. Since the wavelength range coincides with a read-out node of CCD2, we believe this feature is instrumental in nature. If we perform the same analysis on an earlier observation of Sérsic 159-03 the discrepancy disappears, which supports our view. In Peterson et al. (2003) some other RGS observations of clusters of galaxies show the same problem, namely Hydra A, Abell 496 and MKW 3s. A large study of many RGS observations shows that about $\sim 5 \%$ of the observations show an anomaly in the count rate of CCD2 (A. Pollock, priv. comm.). We therefore believe the feature we observe is instrumental in nature and we ignore the 29-33 $\AA$ interval in our analysis.

\subsubsection{Integrated spectrum within $4^{\prime}$}

In Table 4 we show the results from fits to a spectrum extracted from a circular region which has a radius of $4^{\prime}$ and is centred on the core. In this circular region the cluster signal is well above the background. We can use the high signal-to-noise ratio of this spectrum to obtain accurate values for all the fitted parameters. The $\chi^{2}$ value of the single-temperature fit $(1228 / 916)$ indicates that a single-temperature model is potentially not the best description of the data. Multi-temperature models, however, do produce a $\chi_{r}^{2}$ close to 1.0 . Hence, our abundance analysis will be based on the multi-temperature models. There are other models that fit the data equally well. These alternative models will be discussed in Sect. 4.3. The potential presence of a relatively small contribution of other emission components however is of no consequence for the observed trends we present in this section.

An example of a spectrum fitted with a DEM model is shown in Fig. 3. When we set the line emission to zero in our model and plot the residuals, we obtain the plot shown in Fig. 4. The lines from all elements for which we fit abundances are evident in the spectrum. Especially the iron lines are very strong, but also silicon, sulfur and calcium have a high signal-to-noise. Oxygen,
Table 4. Fit results for an EPIC spectrum extracted from a circle with a radius of $4^{\prime}$ and centred on the core. Fluxes are calculated over the $0.3-10 \mathrm{keV}$ range and presented in $10^{-10} \mathrm{erg} \mathrm{cm}^{-2} \mathrm{~s}^{-1} \mathrm{deg}^{-2}$. Emission measure $\left(Y_{\text {thermal }}=\int n_{\mathrm{e}} n_{\mathrm{H}} \mathrm{d} V\right)$ is given in $10^{66} \mathrm{~cm}^{-3}$ and $Y_{\text {pow }}$ is given in $10^{51} \mathrm{ph} \mathrm{s}^{-1} \mathrm{keV}^{-1}$ at $1 \mathrm{keV}$.

\begin{tabular}{lccc}
\hline \hline Parameter & single-temp & wdem-model & gdem-model \\
\hline$Y_{\text {thermal }}$ & $20.31 \pm 0.15$ & $20.55 \pm 0.15$ & $21.07 \pm 0.15$ \\
$F_{\text {thermal }}$ & $19.26 \pm 0.14$ & $19.40 \pm 0.14$ & $19.46 \pm 0.15$ \\
$k T$ & $2.568 \pm 0.009$ & & $2.472 \pm 0.010$ \\
$k T_{\text {mean }}$ & & $2.60 \pm 0.03$ & \\
$k T_{\max }$ & & $3.41 \pm 0.03$ & \\
$\alpha$ & & $0.45 \pm 0.02$ & \\
$\sigma_{T}$ & & & $0.226 \pm 0.005$ \\
$\mathrm{O}$ & $0.36 \pm 0.05$ & $0.30 \pm 0.04$ & $0.19 \pm 0.03$ \\
$\mathrm{Ne}$ & $0.89 \pm 0.08$ & $0.16 \pm 0.08$ & $0.000 \pm 0.013$ \\
$\mathrm{Mg}$ & $0.02 \pm 0.04$ & $0.11 \pm 0.04$ & $0.08 \pm 0.04$ \\
$\mathrm{Si}$ & $0.196 \pm 0.017$ & $0.239 \pm 0.017$ & $0.252 \pm 0.018$ \\
$\mathrm{~S}$ & $0.122 \pm 0.017$ & $0.18 \pm 0.02$ & $0.20 \pm 0.02$ \\
$\mathrm{Ar}$ & & $0.14 \pm 0.04$ & $0.19 \pm 0.05$ \\
$\mathrm{Ca}$ & $0.31 \pm 0.05$ & $0.36 \pm 0.05$ & $0.44 \pm 0.06$ \\
$\mathrm{Fe}$ & $0.360 \pm 0.007$ & $0.346 \pm 0.008$ & $0.242 \pm 0.005$ \\
$\mathrm{Ni}$ & $0.42 \pm 0.09$ & $0.45 \pm 0.08$ & $0.35 \pm 0.08$ \\
$\chi^{2} /$ d.o.f. & $1228 / 916$ & $1017 / 915$ & $949 / 915$ \\
\hline
\end{tabular}

magnesium, argon and nickel are clearly visible: this plot shows the total spectrum; the strengths of these lines are weaker in individual annuli.

The spectral models constrain the abundances very well, except for neon and magnesium, because these lines are blended with iron at this spectral resolution (see Fig. 4). The determination of the oxygen abundance becomes difficult in the outer parts of the cluster. Here, the oxygen in the galactic foreground emission starts to play a more important role and could bias the abundance measurement. In the central region $\left(\lesssim 4^{\prime}\right)$ the cluster flux is high enough to get an accurate measurement. In general, all abundances are between 0.1 and 0.6 times their solar value (Table 4). Only neon shows high values up to 1 , but since the lines of this element are blended with the Fe-L complex, the value is highly correlated with the temperature distribution.

In Fig. 5 we present the total RGS spectrum of Sérsic 159-03 extracted from a $4^{\prime}$ wide strip in the cross-dispersion direction and centred on the core. Because of the high statistics, we can also use the second order spectrum. In both orders the Fe-L spectral line complex between $10-15 \AA$ and the Mg line near $9 \AA$ are well resolved. Above $19 \AA$ the first order spectrum shows a prominent O VIII Ly $\alpha$ line.

The fit results for RGS are shown in Table 5. The elemental abundances determined from the different models are consistent with each other within $3 \sigma$. Unfortunately, the carbon and nitrogen abundances are not well constrained. The width of the oxygen line is consistent with the width of the line profile derived from the continuum emission in MOS1, and the width of the iron lines is about $1 / 3$ smaller (Fig. 6). This suggests that the oxygen distribution is more extended across the cluster centre, while the iron abundance is strongly peaked in the centre. Looking at the $\chi^{2}$ values we see that the fit to the RGS data does not strongly prefer a DEM distribution over a single-temperature model. All models are acceptable, contrary to the merging cluster 2A $0335+096$ described by Werner et al. (2006). 


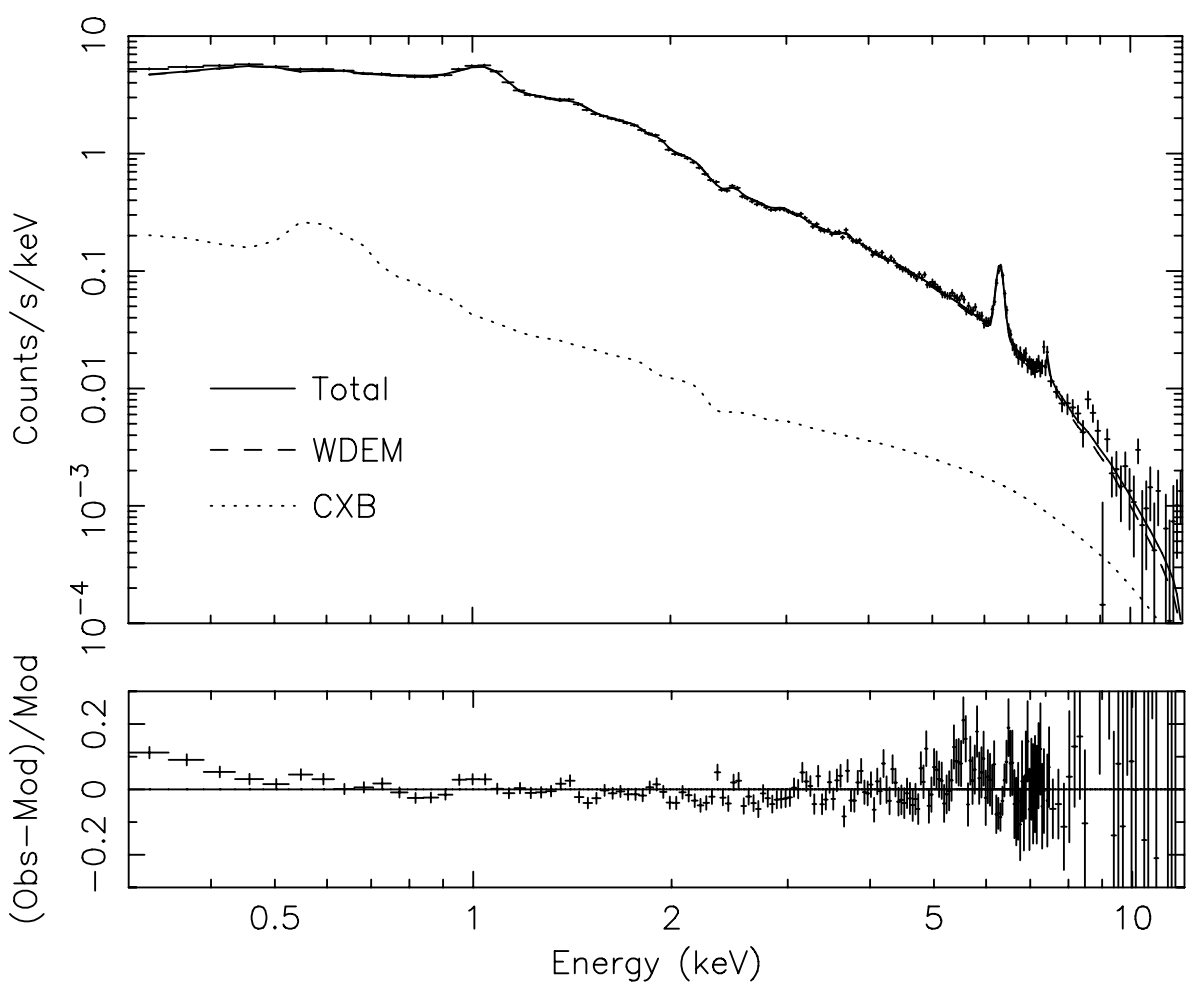

Fig. 3. EPIC spectrum extracted from a circular region of $4^{\prime}$ around the core. The total bestfit model, the wdem model component and the background component are shown.
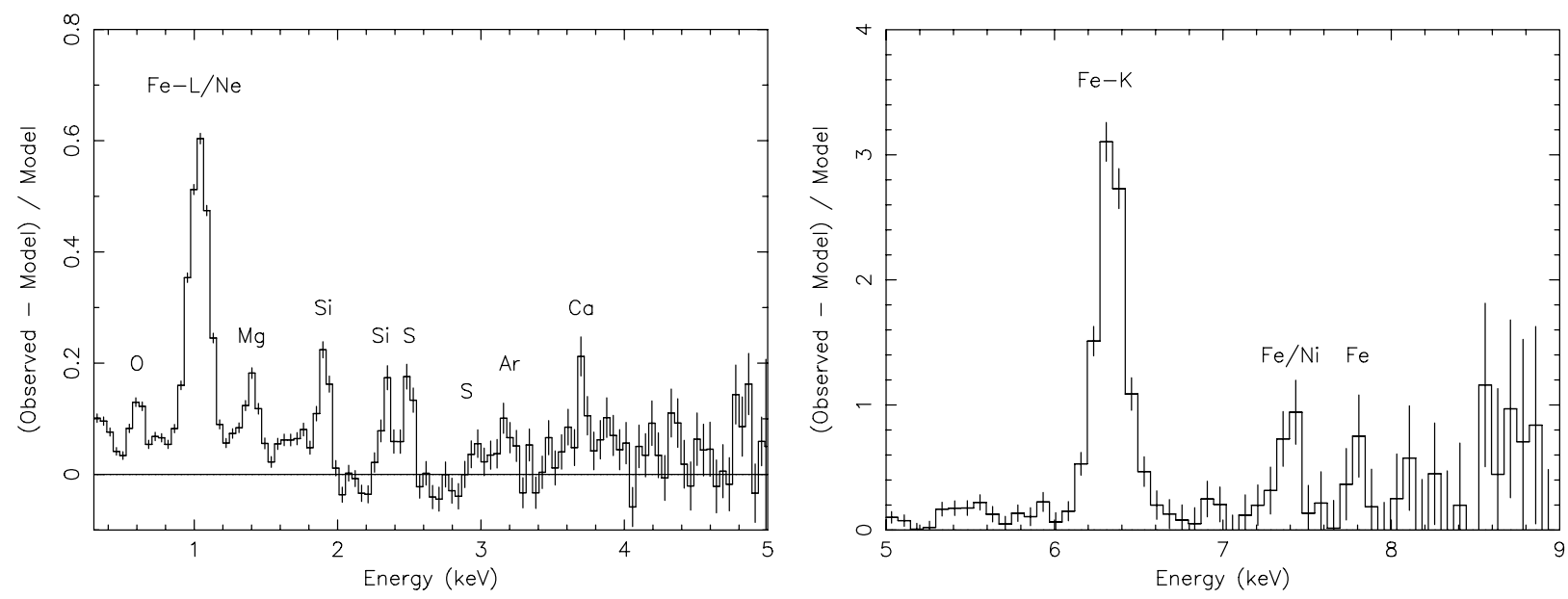

Fig. 4. Residuals of a wdem fit to the EPIC total spectrum extracted from a circle with a radius of $4^{\prime}$. The line emission in the model is set to zero to show the line emission on top of the continuum. The left panel shows the residuals from $0.3-5 \mathrm{keV}$ and the right panel the 5-10 keV range.

\subsubsection{Radial profiles using thermal DEM models}

By fitting the spectra extracted from the annuli for EPIC and from strips for RGS, we can make radial profiles of temperatures and abundances. Again, we fit the spectra with a singletemperature model, a wdem model and a gdem model (Table 6). In Fig. 7 (left panel) we present the temperature profile obtained for these models. For wdem the maximum temperature $\left(k T_{\max }\right)$ and mean temperature $\left(k T_{\text {mean }}\right)$ are shown. The profile shows a slight increase of the temperature within a $2^{\prime}$ radius. Beyond $3^{\prime}$ the temperature rapidly drops to about $1.5 \mathrm{keV}$. The singletemperature profile is consistent with the profile derived from the earlier XMM-Newton observation by Kaastra et al. (2001).

The DEM parameters $\alpha$ (wdem, Fig. 7 middle panel) and $\sigma_{\mathrm{T}}$ (gdem, Fig. 7 right panel) are quite constant, but increase slightly in the outer parts, where the temperature gradient is large. This means that the fit needs a broader range of temperatures to fit the spectrum.

From the fits we obtain radial profiles of the abundances of several elements. The radial profiles of oxygen, silicon, and iron are shown in Fig. 8. We detect a clear decrease of the silicon and iron abundance with radius. For oxygen the situation is less clear: the $\mathrm{O} / \mathrm{Fe}$ ratio in the $0-0.5^{\prime}$ bin is lower than the ratio in the $0.5-4^{\prime}$ interval at $3 \sigma$ confidence level. There is a hint of a decrease of the oxygen abundance like we see for iron, but a flat distribution cannot be excluded.

While silicon is quite well constrained independent of the DEM model used, the iron abundance derived from the gdem model is significantly different from the other two models. The absolute values of the gdem oxygen and iron abundances are consistent with the ones from silicon. The iron abundance from the single-temperature and wdem, however, show a steeper gradient. From these plots it is clear that the used temperature 


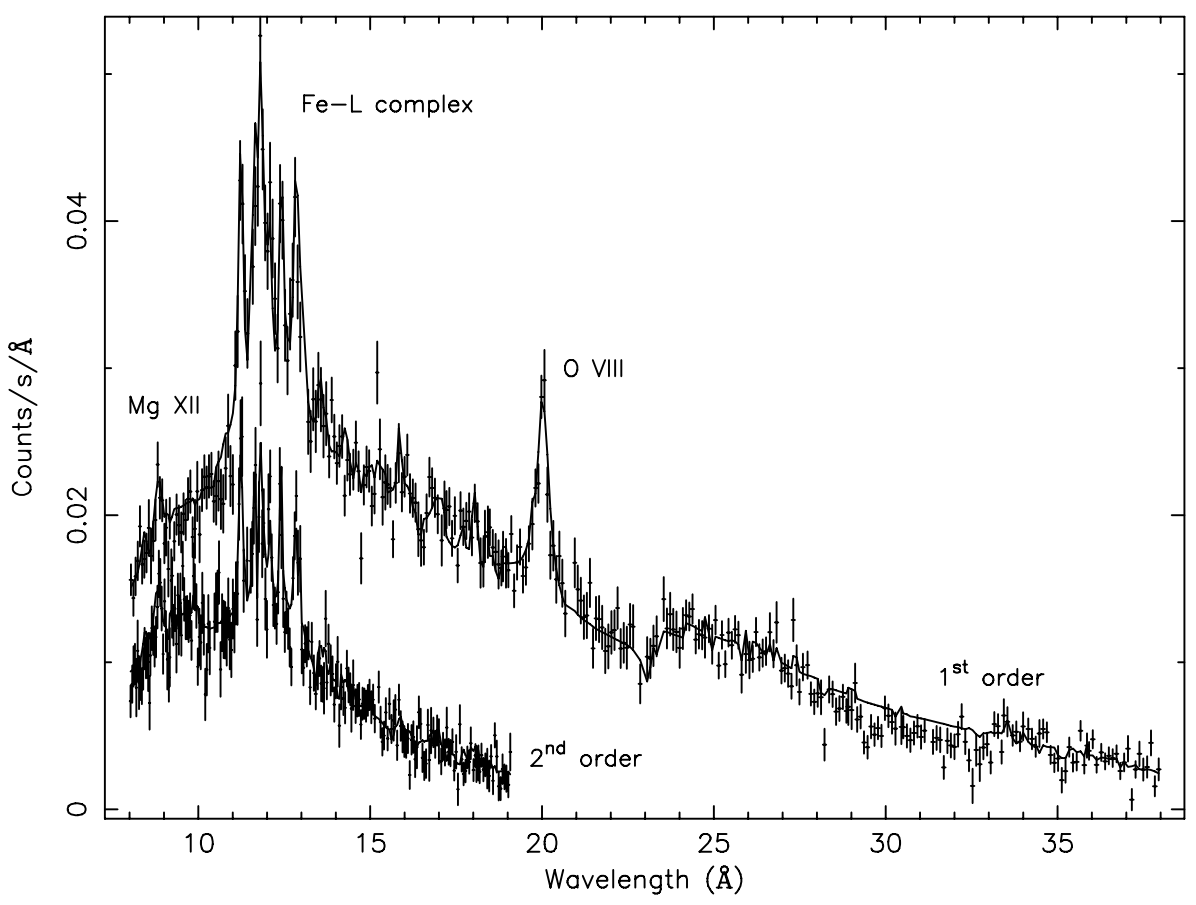

Fig. 5. 1st and 2nd order RGS spectrum of Sérsic 159-03 extracted from a $4^{\prime}$ wide strip centred on the core. The continuous line represents the fitted wdem model. On the $x$-axis we show the observed wavelength.
Table 5. Fit results for RGS spectra extracted from a $4^{\prime}$ wide strip and fitted over a 8-38 ̊̊ range ignoring data from CCD2 (see text). Emission measures $\left(Y=\int n_{\mathrm{e}} n_{\mathrm{H}} \mathrm{d} V\right)$ are given in $10^{66} \mathrm{~cm}^{-3}$. The iron abundance is given with respect to solar abundances. The scale factors for oxygen and iron mentioned below are those explained in Sect. 2.2.

\begin{tabular}{lccc}
\hline \hline Parameter & single-temp & wdem-model & gdem-model \\
\hline$Y$ & $7.27 \pm 0.06$ & $7.08 \pm 0.07$ & $7.25 \pm 0.06$ \\
$k T(\mathrm{keV})$ & $2.6 \pm 0.09$ & & $3.25 \pm 0.18$ \\
$k T_{\text {mean }}(\mathrm{keV})$ & & $3.04 \pm 0.16$ & \\
$k T_{\max }(\mathrm{keV})$ & & $4.0 \pm 0.2$ & \\
$\alpha$ & & $0.46 \pm 0.03$ & \\
$\sigma$ & & & $0.28 \pm 0.02$ \\
$\mathrm{C} / \mathrm{Fe}$ & $0.00 \pm 0.19$ & $0.0 \pm 0.4$ & $0.0 \pm 0.3$ \\
$\mathrm{~N} / \mathrm{Fe}$ & $0.00 \pm 0.18$ & $0.1 \pm 0.5$ & $0.0 \pm 0.5$ \\
$\mathrm{O} / \mathrm{Fe}$ & $0.85 \pm 0.10$ & $0.87 \pm 0.10$ & $0.91 \pm 0.13$ \\
$\mathrm{Ne} / \mathrm{Fe}$ & $1.04 \pm 0.15$ & $0.73 \pm 0.12$ & $0.71 \pm 0.14$ \\
$\mathrm{Mg} / \mathrm{Fe}$ & $0.32 \pm 0.11$ & $0.35 \pm 0.11$ & $0.32 \pm 0.12$ \\
$\mathrm{Fe}$ & $0.98 \pm 0.06$ & $1.20 \pm 0.08$ & $1.14 \pm 0.09$ \\
$\mathrm{Scale}(\mathrm{O})$ & $1.1 \pm 0.2$ & $1.6 \pm 0.3$ & $1.5 \pm 0.3$ \\
$\mathrm{Scale}(\mathrm{Fe})$ & $0.35 \pm 0.05$ & $0.36 \pm 0.05$ & $0.31 \pm 0.05$ \\
$\chi^{2} /$ d.o.f. & $997 / 856$ & $925 / 854$ & $963 / 855$ \\
\hline & & & \\
\hline
\end{tabular}

distribution is very important when measuring elemental abundances.

An overview of all the fitted parameters is given in Table 6 . Apart from the elements we discussed in the previous paragraph, only sulfur is reasonably well constrained up to $9^{\prime}$. In the outermost spatial bin, however, the abundance increases to unphysical values. In the core region sulfur shows the same profile as silicon. The other elements are less well constrained. Neon is always difficult to measure at CCD resolution because it is blended with the Fe-L complex near $1 \mathrm{keV}$. Calcium and nickel are only constrained in the core, but the derived values are not affected by systematics due to different DEM models. In every radial bin the values are consistent with each other. The magnesium and argon abundances are poorly determined. Only in the core region some points are measured at a significance larger than $2 \sigma$. In general

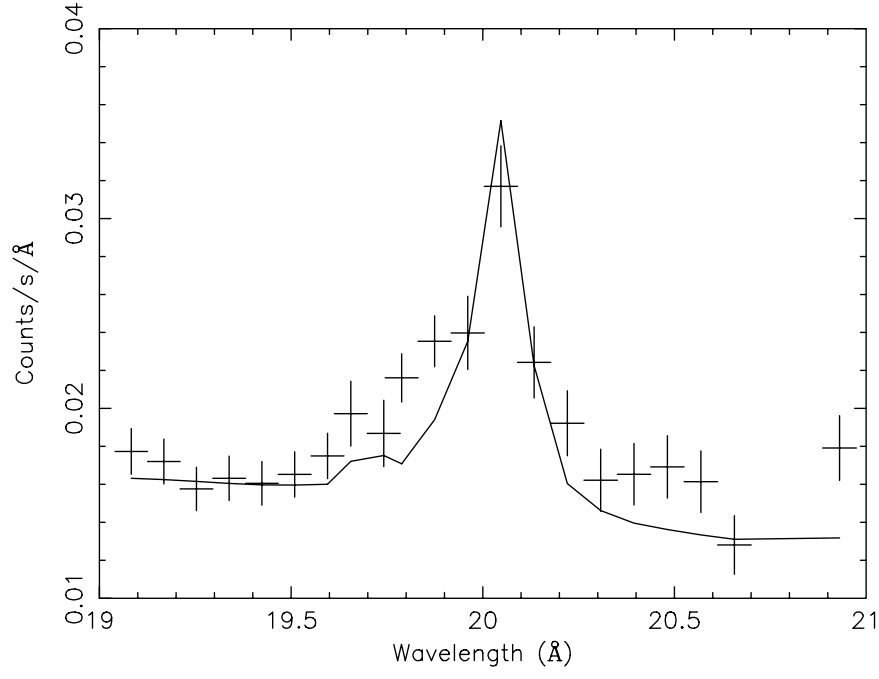

Fig. 6. RGS spectrum in the 19-21 $\AA$ band featuring the $\mathrm{O}$ VIII Ly- $\alpha$ line. The data points represent the measured spectrum, while the model line shows the line when the scale factor is frozen to $\sim 0.3$, which is the best-fit value for the iron lines.

these two elements have upper limits of about 0.2 times the solar abundances.

The $\chi^{2}$ values for the three models are also shown in Table 6 . In the core region all fits are acceptable when we use the condition $\chi_{r}^{2}<1.5$. However, the $\chi_{r}^{2}$ for the single temperature model in the core is much less than in Werner et al. (2006) in the cluster 2A $0335+096$. In the outer parts multi-temperature fitting still results in a better fit, but we do not see significant differences between the models.

From RGS we also extract rectangular regions in the crossdispersion direction of the instrument. The regions we use are defined in Sect. 2.2. Because the cluster does not show significant spatial asymmetries in the cross-dispersion direction, we add the spectra extracted from regions with equal distance to the 
Table 6. Fit results for spatially resolved EPIC spectra. The fitted models are single-temperature CIE (s), wdem (w) and a Gaussian DEM model $(\mathrm{g})$. Fluxes are calculated over the $0.3-10 \mathrm{keV}$ range and are given in unit erg s $\mathrm{cm}^{-2} \mathrm{deg}^{-2}$. Emission measures $\left(Y=\int n_{\mathrm{e}} n_{\mathrm{H}} \mathrm{d} V\right)$ are given in $10^{66} \mathrm{~cm}^{-3}$.

\begin{tabular}{|c|c|c|c|c|c|c|c|c|}
\hline & $\mathrm{M}$ & $0-0.5^{\prime}$ & $0.5-1.0^{\prime}$ & $1.0-2.0^{\prime}$ & $2.0-3.0^{\prime}$ & $3.0-4.0^{\prime}$ & $4.0-6.0^{\prime}$ & $6.0-9.0^{\prime}$ \\
\hline \multirow[t]{3}{*}{$Y$} & $\mathrm{~s}$ & $4.75 \pm 0.05$ & $5.55 \pm 0.05$ & $6.17 \pm 0.05$ & $2.88 \pm 0.03$ & $1.40 \pm 0.02$ & $1.62 \pm 0.04$ & $1.21 \pm 0.04$ \\
\hline & $\mathrm{w}$ & $4.72 \pm 0.05$ & $5.58 \pm 0.05$ & $6.23 \pm 0.05$ & $2.91 \pm 0.03$ & $1.42 \pm 0.02$ & $1.62 \pm 0.03$ & $1.21 \pm 0.03$ \\
\hline & $\mathrm{g}$ & $4.86 \pm 0.04$ & $5.72 \pm 0.05$ & $6.36 \pm 0.05$ & $2.97 \pm 0.03$ & $1.44 \pm 0.02$ & $1.55 \pm 0.03$ & $0.94 \pm 0.04$ \\
\hline \multirow[t]{3}{*}{$F$} & $\mathrm{~s}$ & $(2.59 \pm 0.02) \times 10^{-8}$ & $(1.038 \pm 0.009) \times 10^{-8}$ & $(2.76 \pm 0.02) \times 10^{-9}$ & $(7.72 \pm 0.09) \times 10^{-10}$ & $(3.17 \pm 0.05) \times 10^{-10}$ & $(1.08 \pm 0.02) \times 10^{-10}$ & $(2.59 \pm 0.08) \times 10^{-11}$ \\
\hline & $\mathrm{w}$ & $(2.60 \pm 0.03) \times 10^{-8}$ & $(1.042 \pm 0.010) \times 10^{-8}$ & $(2.77 \pm 0.02) \times 10^{-9}$ & $(7.79 \pm 0.09) \times 10^{-10}$ & $(3.20 \pm 0.05) \times 10^{-10}$ & $(1.093 \pm 0.019) \times 10^{-10}$ & $(2.59 \pm 0.08) \times 10^{-11}$ \\
\hline & $\mathrm{g}$ & $(2.61 \pm 0.02) \times 10^{-8}$ & $(1.047 \pm 0.010) \times 10^{-8}$ & $(2.78 \pm 0.02) \times 10^{-9}$ & $(7.84 \pm 0.08) \times 10^{-10}$ & $(3.24 \pm 0.05) \times 10^{-10}$ & $(1.112 \pm 0.018) \times 10^{-10}$ & $(2.59 \pm 0.09) \times 10^{-11}$ \\
\hline$k T$ & $\mathrm{~s}$ & $2.353 \pm 0.012$ & $2.557 \pm 0.013$ & $2.640 \pm 0.015$ & $2.64 \pm 0.03$ & $2.50 \pm 0.04$ & $2.21 \pm 0.05$ & $1.41 \pm 0.04$ \\
\hline$(\mathrm{keV})$ & $\mathrm{g}$ & $2.280 \pm 0.011$ & $2.471 \pm 0.013$ & $2.547 \pm 0.015$ & $2.57 \pm 0.02$ & $2.48 \pm 0.04$ & $2.40 \pm 0.05$ & $1.83 \pm 0.09$ \\
\hline$k T_{\text {mean }}$ & w & $2.39 \pm 0.03$ & $2.59 \pm 0.05$ & $2.67 \pm 0.05$ & $2.69 \pm 0.08$ & $2.56 \pm 0.12$ & $2.27 \pm 0.11$ & \\
\hline$k T_{\max }$ & w & $3.01 \pm 0.03$ & $3.27 \pm 0.04$ & $3.42 \pm 0.05$ & $3.51 \pm 0.08$ & $3.31 \pm 0.13$ & $2.90 \pm 0.12$ & $1.42 \pm 0.08$ \\
\hline$\alpha$ & w & $0.350 \pm 0.019$ & $0.36 \pm 0.03$ & $0.39 \pm 0.03$ & $0.44 \pm 0.05$ & $0.42 \pm 0.07$ & $0.38 \pm 0.07$ & $0.01 \pm 0.09$ \\
\hline$\sigma_{\mathrm{T}}$ & $\mathrm{g}$ & $0.203 \pm 0.005$ & $0.208 \pm 0.007$ & $0.209 \pm 0.008$ & $0.236 \pm 0.012$ & $0.244 \pm 0.019$ & $0.24 \pm 0.02$ & $0.14 \pm 0.04$ \\
\hline \multirow[t]{3}{*}{$\mathrm{O} / \mathrm{Fe}$} & $\mathrm{s}$ & $0.76 \pm 0.12$ & $1.12 \pm 0.13$ & $1.17 \pm 0.17$ & $1.3 \pm 0.3$ & $1.2 \pm 0.4$ & $0.2 \pm 1.0$ & $0.0 \pm 1.7$ \\
\hline & w & $0.76 \pm 0.11$ & $1.05 \pm 0.14$ & $1.06 \pm 0.17$ & $1.2 \pm 0.3$ & $1.1 \pm 0.4$ & $0.4 \pm 0.6$ & $0.0 \pm 1.7$ \\
\hline & g & $0.77 \pm 0.10$ & $1.05 \pm 0.13$ & $1.05 \pm 0.17$ & $1.0 \pm 0.3$ & $0.4 \pm 0.4$ & $0.00 \pm 0.05$ & $0.00 \pm 0.06$ \\
\hline \multirow[t]{3}{*}{$\mathrm{Ne} / \mathrm{Fe}$} & $\mathrm{s}$ & $2.6 \pm 0.2$ & $2.0 \pm 0.2$ & $1.4 \pm 0.3$ & $1.4 \pm 0.4$ & $1.5 \pm 0.6$ & $1.7 \pm 1.1$ & $0.0 \pm 0.9$ \\
\hline & w & $0.9 \pm 0.2$ & $0.4 \pm 0.3$ & $0.00 \pm 0.15$ & $0.0 \pm 0.2$ & $0.0 \pm 0.5$ & $0.2 \pm 0.9$ & $0.0 \pm 0.9$ \\
\hline & $\mathrm{g}$ & $0.00 \pm 0.16$ & $0.00 \pm 0.07$ & $0.00 \pm 0.05$ & $0.00 \pm 0.09$ & $0.0 \pm 0.2$ & $0.0 \pm 0.5$ & $0.0 \pm 0.7$ \\
\hline \multirow[t]{3}{*}{$\mathrm{Mg} / \mathrm{Fe}$} & $\mathrm{s}$ & $0.10 \pm 0.10$ & $0.00 \pm 0.12$ & $0.00 \pm 0.08$ & $0.01 \pm 0.19$ & $0.00 \pm 0.09$ & $0.1 \pm 0.5$ & $0.0 \pm 0.4$ \\
\hline & w & $0.29 \pm 0.10$ & $0.18 \pm 0.13$ & $0.13 \pm 0.15$ & $0.3 \pm 0.3$ & $0.00 \pm 0.11$ & $0.3 \pm 0.5$ & $0.0 \pm 0.4$ \\
\hline & $\mathrm{g}$ & $0.34 \pm 0.14$ & $0.20 \pm 0.18$ & $0.1 \pm 0.2$ & $0.5 \pm 0.4$ & $0.0 \pm 0.2$ & $1.3 \pm 0.6$ & $0.0 \pm 0.7$ \\
\hline \multirow[t]{3}{*}{$\mathrm{Si} / \mathrm{Fe}$} & $\mathrm{s}$ & $0.58 \pm 0.05$ & $0.45 \pm 0.06$ & $0.57 \pm 0.07$ & $0.38 \pm 0.12$ & $0.43 \pm 0.09$ & $0.8 \pm 0.3$ & $0.5 \pm 0.5$ \\
\hline & $\mathrm{w}$ & $0.69 \pm 0.05$ & $0.56 \pm 0.06$ & $0.70 \pm 0.08$ & $0.51 \pm 0.13$ & $0.5 \pm 0.2$ & $0.8 \pm 0.2$ & $0.4 \pm 0.5$ \\
\hline & $\mathrm{g}$ & $1.07 \pm 0.08$ & $0.88 \pm 0.09$ & $1.08 \pm 0.12$ & $0.8 \pm 0.2$ & $0.9 \pm 0.3$ & $1.4 \pm 0.4$ & $0.7 \pm 0.6$ \\
\hline \multirow[t]{3}{*}{$\mathrm{S} / \mathrm{Fe}$} & $\mathrm{s}$ & $0.43 \pm 0.06$ & $0.34 \pm 0.07$ & $0.19 \pm 0.09$ & $0.22 \pm 0.16$ & $0.3 \pm 0.3$ & $0.97 \pm 0.17$ & $5.6 \pm 1.7$ \\
\hline & w & $0.56 \pm 0.06$ & $0.47 \pm 0.08$ & $0.35 \pm 0.09$ & $0.40 \pm 0.15$ & $0.5 \pm 0.2$ & $1.0 \pm 0.4$ & $5.6 \pm 1.8$ \\
\hline & $\mathrm{g}$ & $0.90 \pm 0.09$ & $0.76 \pm 0.11$ & $0.57 \pm 0.13$ & $0.7 \pm 0.2$ & $0.9 \pm 0.4$ & $1.3 \pm 0.5$ & $3.5 \pm 1.2$ \\
\hline \multirow[t]{3}{*}{$\mathrm{Ar} / \mathrm{Fe}$} & $\mathrm{s}$ & $0.02 \pm 0.13$ & $0.20 \pm 0.17$ & $0.4 \pm 0.2$ & $0.0 \pm 0.4$ & $0.6 \pm 0.7$ & $1.9 \pm 1.1$ & $16.8 \pm 6.4$ \\
\hline & w & $0.19 \pm 0.14$ & $0.39 \pm 0.18$ & $0.7 \pm 0.2$ & $0.2 \pm 0.4$ & $0.8 \pm 0.7$ & $1.8 \pm 1.1$ & $17.0 \pm 6.4$ \\
\hline & $\mathrm{g}$ & $0.4 \pm 0.2$ & $0.7 \pm 0.3$ & $1.2 \pm 0.4$ & $0.4 \pm 0.7$ & $1.4 \pm 1.2$ & $1.6 \pm 1.5$ & $5.4 \pm 3.5$ \\
\hline \multirow[t]{3}{*}{$\mathrm{Ca} / \mathrm{Fe}$} & $\mathrm{s}$ & $0.59 \pm 0.18$ & $1.1 \pm 0.2$ & $1.1 \pm 0.3$ & $1.0 \pm 0.5$ & $1.6 \pm 1.0$ & $3.6 \pm 1.5$ & $36.6 \pm 15.6$ \\
\hline & w & $0.67 \pm 0.18$ & $1.3 \pm 0.2$ & $1.2 \pm 0.3$ & $1.0 \pm 0.6$ & $1.6 \pm 1.0$ & $3.0 \pm 1.6$ & $36.2 \pm 15.9$ \\
\hline & $\mathrm{g}$ & $1.2 \pm 0.3$ & $2.3 \pm 0.4$ & $2.2 \pm 0.5$ & $1.7 \pm 1.1$ & $2.2 \pm 1.8$ & $0.4 \pm 2.3$ & $0.0 \pm 6.5$ \\
\hline \multirow[t]{3}{*}{$\mathrm{Fe}$} & $\mathrm{s}$ & $0.453 \pm 0.012$ & $0.381 \pm 0.010$ & $0.294 \pm 0.009$ & $0.239 \pm 0.011$ & $0.209 \pm 0.013$ & $0.14 \pm 0.02$ & $0.049 \pm 0.009$ \\
\hline & w & $0.458 \pm 0.011$ & $0.371 \pm 0.011$ & $0.282 \pm 0.009$ & $0.224 \pm 0.012$ & $0.196 \pm 0.017$ & $0.140 \pm 0.016$ & $0.049 \pm 0.009$ \\
\hline & $\mathrm{g}$ & $0.314 \pm 0.007$ & $0.253 \pm 0.007$ & $0.191 \pm 0.007$ & $0.148 \pm 0.008$ & $0.134 \pm 0.012$ & $0.134 \pm 0.012$ & $0.13 \pm 0.02$ \\
\hline \multirow[t]{3}{*}{$\mathrm{Ni} / \mathrm{Fe}$} & $\mathrm{s}$ & $1.0 \pm 0.2$ & $0.9 \pm 0.2$ & $0.0 \pm 0.3$ & $0.0 \pm 0.2$ & $0.3 \pm 0.8$ & $0.0 \pm 0.3$ & $0.0 \pm 2.3$ \\
\hline & w & $1.2 \pm 0.2$ & $1.0 \pm 0.3$ & $0.2 \pm 0.3$ & $0.0 \pm 0.5$ & $1.1 \pm 1.0$ & $0.0 \pm 0.3$ & $0.0 \pm 2.4$ \\
\hline & $\mathrm{g}$ & $1.4 \pm 0.3$ & $1.1 \pm 0.4$ & $0.0 \pm 0.5$ & $0.0 \pm 0.8$ & $2.8 \pm 1.5$ & $0.3 \pm 1.6$ & $6.0 \pm 2.5$ \\
\hline \multirow{3}{*}{$\chi^{2} /$ d.o.f. } & $\mathrm{s}$ & $1213 / 829$ & 1084 / 846 & $1240 / 852$ & $1191 / 804$ & $1270 / 772$ & $1113 / 777$ & $1045 / 757$ \\
\hline & $\mathrm{w}$ & 1045 / 828 & $969 / 845$ & $1136 / 851$ & 1109 / 803 & $1218 / 771$ & $1095 / 776$ & $1045 / 756$ \\
\hline & $\mathrm{g}$ & $981 / 828$ & $890 / 845$ & 1083 / 851 & 1034 / 803 & $1131 / 771$ & $1042 / 776$ & $1079 / 756$ \\
\hline
\end{tabular}

dispersion axis: region $1+5$ and $2+4$ as defined in the lower panel of Fig. 2. This way we are able to derive radial profiles of the core up to a radius of $2^{\prime}$. The best fit values for three models are presented in Table 7. The fit results show signs of a temperature decrement in the core. However, the temperatures we determine from RGS are systematically higher than those from EPIC. This is not surprising, because the RGS spectrum also contains emission from the hot gas just outside $2^{\prime}$ which falls within the rectangular field-of-view. From the wdem model fits we see that the value of $\alpha$ increases with radius, while the width of the Gaussian DEM distribution shows a drop in the 1.0-2.0' bin. As expected from the EPIC profiles, the $\mathrm{O} / \mathrm{Fe}$ ratio is lower in the core than in the outer parts. The $\mathrm{Ne} / \mathrm{Fe}$ ratio, however, is consistent with being flat within $2^{\prime}$ from the core. Finally, the width of the lines, indicated by the scale parameter (see Sect. 2.2), increases outside the core. There it is consistent with being 1.0. In these models we fit the average of all the line widths with respect to the continuum surface brightness.
From the $\mathrm{O}$ and $\mathrm{Fe}$ abundances measured with EPIC and RGS in the inner $2^{\prime}$ of the cluster, we detect a jump in the $\mathrm{O} / \mathrm{Fe}$ ratios between the inner $0.5^{\prime}$ and the annulus from $0.5-2.0^{\prime}$. To illustrate this, we combine the singletemperature EPIC and RGS results in the $0.5-2.0^{\prime}$ region. From EPIC we obtain $(\mathrm{O} / \mathrm{Fe})_{0.5^{\prime}-2.0^{\prime}}=1.14 \pm 0.10$ which is significantly higher than the central $(\mathrm{O} / \mathrm{Fe})_{0.0^{\prime}-0.5^{\prime}}=0.76 \pm$ 0.12 . The combined $\mathrm{O} / \mathrm{Fe}$ ratio from the $0.5-2.0^{\prime} \mathrm{RGS}$ results is also significantly higher, $(\mathrm{O} / \mathrm{Fe})_{0.5^{\prime}-2.0^{\prime}}=0.84 \pm 0.09$, compared to the central value $(\mathrm{O} / \mathrm{Fe})_{0.0^{\prime}-0.5^{\prime}}=0.53 \pm 0.05$. This jump has a confidence of $2.5 \sigma$ and $3 \sigma$ in EPIC and RGS respectively, which corresponds to a combined significance of $3.9 \sigma$. Despite the fact that the absolute values of the $\mathrm{O} / \mathrm{Fe}$ ratio are different for the EPIC and RGS results, the relative increase of the $\mathrm{O} / \mathrm{Fe}$ ratio is the same for both instruments: $1.5 \pm 0.3$ (EPIC) and $1.6 \pm 0.2$ (RGS). In the wdem and gdem results the relative jump in $\mathrm{O} / \mathrm{Fe}$ is equal or lower, but still consistent with the result for the single temperature 

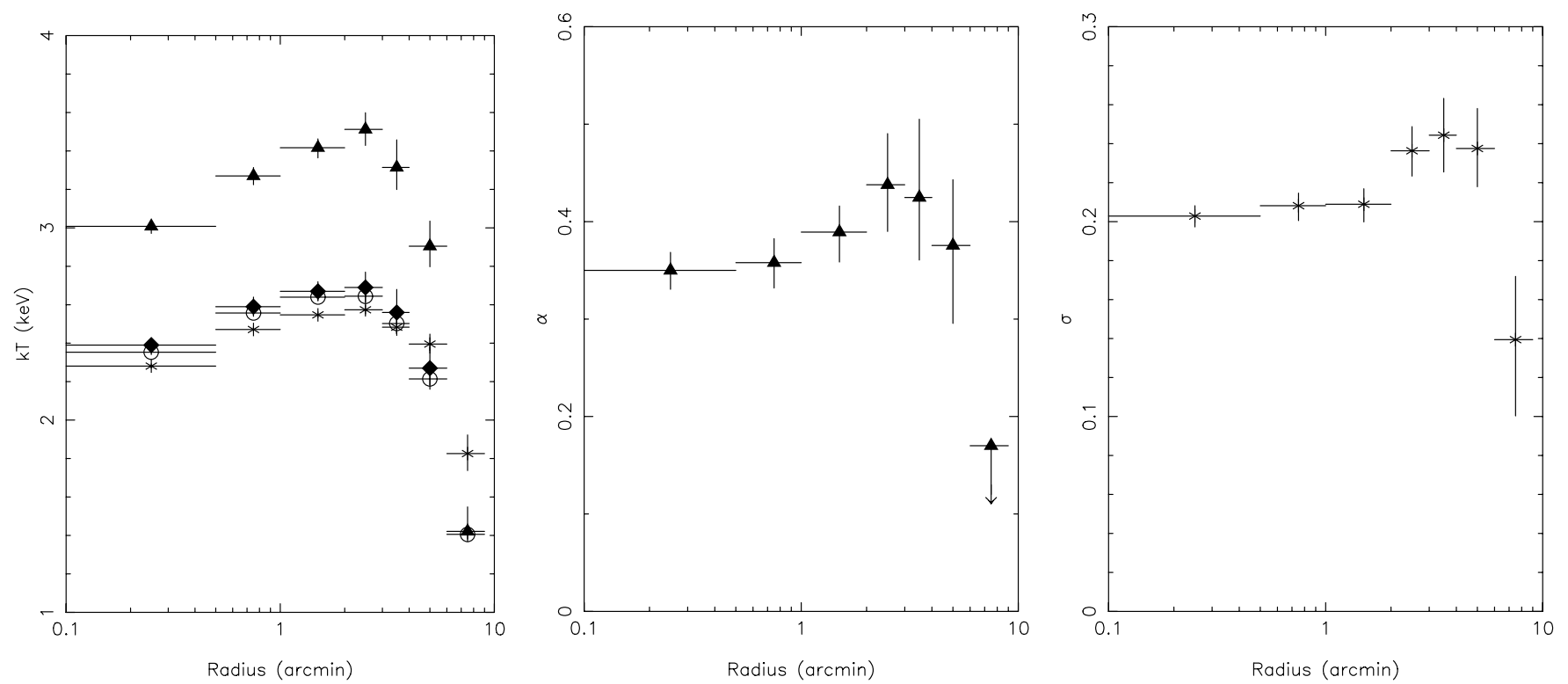

Fig. 7. DEM model fit results for the three models: single-temperature $(\bigcirc)$, wdem $(\mathbf{\Lambda})$ and $\operatorname{gdem}(*)$. In the left panel we show the radial temperature profiles for all models, including the $k T_{\max }(\mathbf{\Lambda})$ and $k T_{\text {mean }}(\downarrow)$ from the wdem parameters. In the middle and right panel we show the radial profiles of the DEM parameters $\alpha$ and $\sigma_{\mathrm{T}}$.
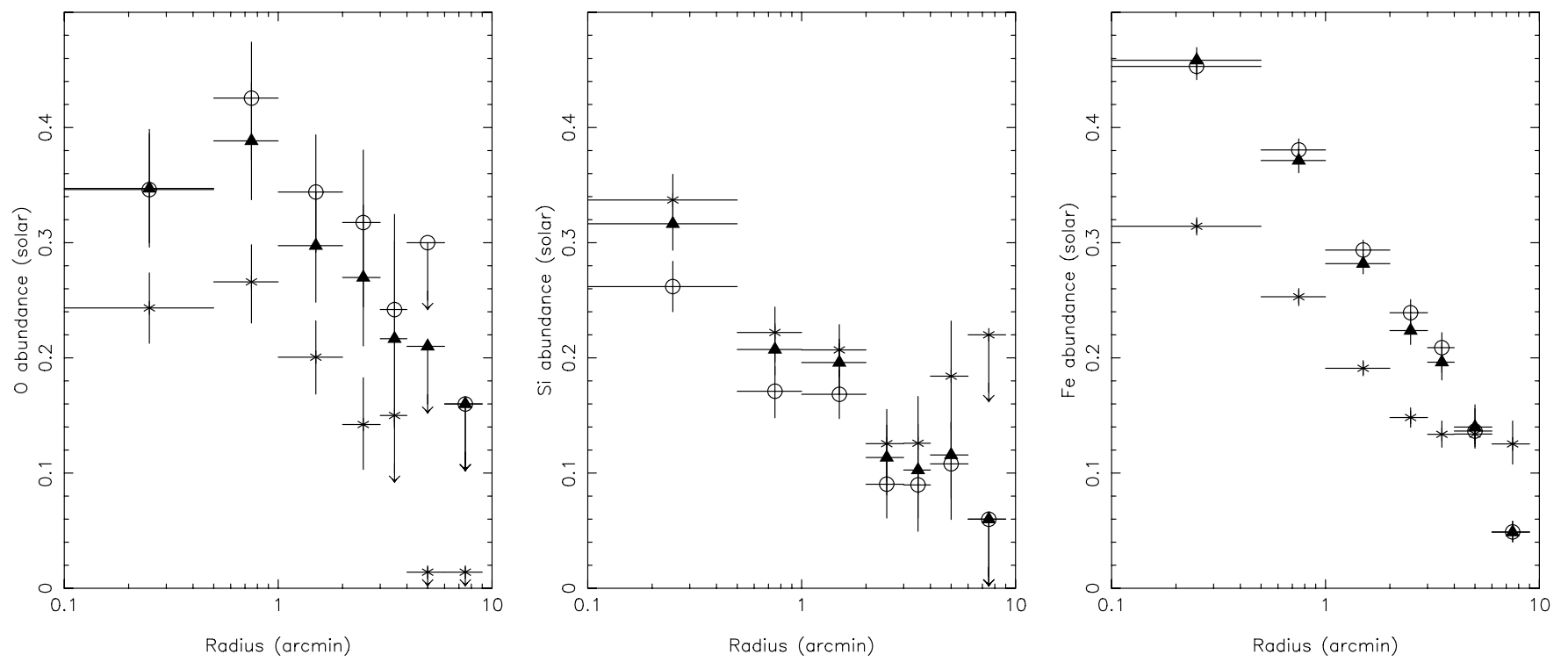

Fig. 8. Abundance profiles of oxygen, silicon and iron derived using three models: single-temperature $(\bigcirc)$, wdem $(\mathbf{\Lambda})$ and $g \operatorname{dem}(*)$.

model: $1.4 \pm 0.3(\mathrm{EPIC} /$ wdem $), 1.4 \pm 0.2(\mathrm{EPIC} /$ gdem $), 1.6 \pm$ $0.3(\mathrm{RGS} /$ wdem $)$ and $1.5 \pm 0.3(\mathrm{RGS} /$ gdem $)$.

\subsection{Abundances and SNIa/SNII/Population-III models}

From the single-temperature (CIE) and DEM models we fit to the data, we obtain the abundances of the elements for which line emission is detected. Assuming that all the elements originate from SNIa, SNII and PopIII stars, we can construct a simple model to obtain the relative contribution of these objects to the enrichment of the ICM.

We use several SNIa yields obtained from two physically different models adapted from Iwamoto et al. (1999) to fit our abundances. The W7 model describes a so-called slow deflagration model, while the WDD2 is calculated using delayed-detonation (DD) models, which is the currently favoured type Ia explosion scenario. Note that with SNII we mean all types of core-collapse supernovae including types Ib and Ic. We use the SNII yields integrated over the stellar population calculated by Tsujimoto et al. (1995) and Iwamoto et al. (1999). For the PopIII-star SN yields we use two models from Heger \& Woosley (2002) with different core masses of the PopIII star: $65 M_{\odot}$ and $130 M_{\odot}$. These two masses are the lowest and the highest core mass considered in Heger \& Woosley (2002).

For every element $i$ the total number of particles $N_{i}$ is a linear combination of the number of atoms produced by supernova type Ia $\left(\mathrm{Y}_{i, \mathrm{Ia}}\right)$, type II $\left(\mathrm{Y}_{i, \mathrm{II}}\right)$ and PopIII stars $\left(\mathrm{Y}_{i, \mathrm{III}}\right)$.

$N_{i}=a \mathrm{Y}_{i, \mathrm{Ia}}+b \mathrm{Y}_{i, \mathrm{II}}+c \mathrm{Y}_{i, \mathrm{III}}$,

where $a, b$ and $c$ are multiplication factors of SNIa, SNII, and PopIII stars respectively. The total number of particles for an element can be easily converted into a number abundance. This reduces to a system of three variables $(a, b$ an $c)$ and nine data points $(\mathrm{O}, \mathrm{Ne}, \mathrm{Mg}, \mathrm{Si}, \mathrm{S}, \mathrm{Ar}, \mathrm{Ca}, \mathrm{Fe}$ and $\mathrm{Ni})$. We present 
Table 7. Fit results for spatially resolved RGS spectra between $8-38 \AA$ excluding CCD2. The fitted models are single-temperature CIE (s), $w d e m(\mathrm{w})$ and a Gaussian DEM model $(\mathrm{g})$. Emission measures $(Y=$ $\int n_{\mathrm{e}} n_{\mathrm{H}} \mathrm{d} V$ ) are given in $10^{66} \mathrm{~cm}^{-3}$. The iron abundance is fixed to 1.0 with respect to solar abundances.

\begin{tabular}{lcccc}
\hline \hline Parameter & Mod & $0-0.5^{\prime}$ & $0.5-1.0^{\prime}$ & $1.0-2.0^{\prime}$ \\
\hline$Y$ & $\mathrm{~s}$ & $3.69 \pm 0.04$ & $1.80 \pm 0.04$ & $1.46 \pm 0.03$ \\
& $\mathrm{w}$ & $3.60 \pm 0.04$ & $1.78 \pm 0.04$ & $1.30 \pm 0.05$ \\
$k T$ & $\mathrm{~g}$ & $3.74 \pm 0.04$ & $1.88 \pm 0.05$ & $1.46 \pm 0.04$ \\
$(\mathrm{keV})$ & $\mathrm{s}$ & $3.23 \pm 0.09$ & $4.2 \pm 0.2$ & $4.3 \pm 0.3$ \\
$k T_{\text {mean }}$ & $\mathrm{g}$ & $4.09 \pm 0.16$ & $5.1 \pm 0.6$ & $4.3 \pm 0.3$ \\
$k T_{\max }$ & $\mathrm{w}$ & $3.53 \pm 0.14$ & $4.7 \pm 0.4$ & $4.3 \pm 0.5$ \\
$\alpha$ & $\mathrm{w}$ & $4.76 \pm 0.18$ & $6.6 \pm 0.5$ & $6.5 \pm 0.7$ \\
$\sigma_{\mathrm{T}}$ & $\mathrm{w}$ & $0.53 \pm 0.04$ & $0.66 \pm 0.09$ & $1.06 \pm 0.18$ \\
$\mathrm{O} / \mathrm{Fe}$ & $\mathrm{g}$ & $0.342 \pm 0.018$ & $0.35 \pm 0.06$ & $0.00 \pm 0.12$ \\
& $\mathrm{~S}$ & $0.53 \pm 0.05$ & $0.77 \pm 0.12$ & $0.96 \pm 0.15$ \\
& $\mathrm{w}$ & $0.51 \pm 0.05$ & $0.72 \pm 0.12$ & $1.2 \pm 0.3$ \\
$\mathrm{Ne} / \mathrm{Fe}$ & $\mathrm{g}$ & $0.54 \pm 0.05$ & $0.68 \pm 0.18$ & $0.93 \pm 0.17$ \\
& $\mathrm{~s}$ & $1.10 \pm 0.12$ & $1.7 \pm 0.3$ & $0.8 \pm 0.4$ \\
$\mathrm{Mg} / \mathrm{Fe}$ & $\mathrm{w}$ & $0.79 \pm 0.11$ & $0.9 \pm 0.3$ & $0.00 \pm 0.10$ \\
& $\mathrm{~g}$ & $0.75 \pm 0.12$ & $0.9 \pm 0.4$ & $0.8 \pm 0.4$ \\
$\mathrm{Scale}$ & $\mathrm{S}$ & $0.16 \pm 0.10$ & $0.00 \pm 0.10$ & $0.00 \pm 0.04$ \\
& $\mathrm{~g}$ & $0.32 \pm 0.11$ & $0.0 \pm 0.2$ & $0.00 \pm 0.12$ \\
& $\mathrm{~g}$ & $0.28 \pm 0.11$ & $0.00 \pm 0.11$ & $0.00 \pm 0.05$ \\
$\chi^{2} /$ d.o.f. & $\mathrm{g}$ & $0.50 \pm 0.08$ & $1.14 \pm 0.17$ & $1.3 \pm 0.3$ \\
& $\mathrm{~g}$ & $0.45 \pm 0.07$ & $1.1 \pm 0.2$ & $2.8 \pm 0.4$ \\
& $\mathrm{w}$ & $1100 / 858 / 855$ & $970 / 858$ & $925 / 858$ \\
& $\mathrm{~g}$ & $1039 / 857$ & $971 / 857$ & $926 / 857$ \\
\hline & & & &
\end{tabular}

the ratio of the relative numbers of SNIa, SNII and PopIII with respect to the total number.

We fit the abundances we obtain from the EPIC results of the core region to a model consisting of SNIa, SNII and PopIII-star yields in order to determine their relative contribution to the ICM. In Table 8 we compare two SNIa yield models fitted together with the SNII and PopIII yield models to the measured abundances in Table 4. We use the abundances derived from the fits with the wdem model. If we include the calcium abundance, none of the fits are statistically acceptable. However, if we ignore the calcium data point, we obtain a $\chi_{r}^{2}$ of about 1 for the WDD2 model. We also test other SNIa models listed in Iwamoto et al. (1999): W70, WDD1, WDD3, CDD1 and CDD2. Their best-fit ratios were in general similar to the WDD2 and W7 models, but with a higher $\chi^{2}$.

The relative contribution of PopIII stars is in all fits smaller than three times its error, thus it is not significantly detected. Because of the large yields per PopIII event, a small number of PopIII stars can in principal contribute a lot to the abundance. The abundance pattern of PopIII stars, however, resemble the patterns of SNIa and SNII depending on the core mass. The SNIa or SNII patterns can mostly compensate for the PopIII contribution, when it is left out in the fit. The $\chi^{2}$ does improve only marginally when we add PopIII star yields to a model containing only SNIa and SNII yields. The best fit using just SNIa and SNII models including calcium gives a $\chi^{2} /$ d.o.f. of $15 / 7$ and a SNIa contribution of $0.35 \pm 0.03$. In fact some models fit the data best by putting a negative value to the PopIII or SNII contribution, which is unphysical. The $65 M_{\odot}$ PopIII model produces a slightly higher PopIII contribution than the one for $130 M_{\odot}$. But since the mass of the expelled material is higher for the latter model, the number of stars needs to be higher in the $65 M_{\odot}$ model to get a comparable effect. The errors on these
Table 8. Relative contribution of SNIa, SNII and PopIII stars to the enrichment of the ICM. We compare two SNIa models in Iwamoto et al. (1999) with the data. We only show results from $w$ dem fits, because we could only get an acceptable fit using these data. The values shown here are the fractions with respect to the sum of all contributions (SNIa + SNII + PopIII)

\begin{tabular}{|c|c|c|c|c|}
\hline Type & \multicolumn{2}{|c|}{$M_{\text {PopIII }}=130 M_{\odot}$} & \multicolumn{2}{|c|}{$M_{\text {PopIII }}=65 M_{\odot}$} \\
\hline & Value & $\chi^{2} /$ d.o.f. & Value & $\chi^{2} /$ d.o.f. \\
\hline \multicolumn{5}{|c|}{ W7 } \\
\hline SNIa & $0.33 \pm 0.09$ & \multirow{3}{*}{$41 / 6$} & $0.30 \pm 0.04$ & \multirow{3}{*}{$55 / 6$} \\
\hline SNII & $0.7 \pm 0.2$ & & $0.70 \pm 0.14$ & \\
\hline PopIII & $(3.4 \pm 1.4) \times 10^{-3}$ & & $(-3 \pm 7) \times 10^{-3}$ & \\
\hline \multicolumn{5}{|c|}{ WDD2 } \\
\hline SNIa & $0.38 \pm 0.08$ & \multirow{3}{*}{$12 / 6$} & $0.48 \pm 0.10$ & \multirow{3}{*}{$12 / 6$} \\
\hline SNII & $0.63 \pm 0.15$ & & $0.5 \pm 0.2$ & \\
\hline PopIII & $(-1.8 \pm 1.2) \times 10^{-3}$ & & $(1.7 \pm 1.1) \times 10^{-2}$ & \\
\hline \multicolumn{5}{|c|}{ Fits with the calcium abundance excluded: } \\
\hline Type & \multicolumn{2}{|c|}{$M_{\text {PopIII }}=130 M_{\odot}$} & \multicolumn{2}{|c|}{$M_{\text {PopIII }}=65 M_{\odot}$} \\
\hline & Value & $\chi^{2} /$ d.o.f. & Value & $\chi^{2} /$ d.o.f. \\
\hline \multicolumn{5}{|c|}{ W7 } \\
\hline SNIa & $0.34 \pm 0.09$ & \multirow{3}{*}{$29 / 5$} & $0.32 \pm 0.05$ & \multirow{3}{*}{$41 / 5$} \\
\hline SNII & $0.7 \pm 0.2$ & & $0.69 \pm 0.15$ & \\
\hline PopIII & $(2.9 \pm 1.3) \times 10^{-3}$ & & $(-2 \pm 7) \times 10^{-3}$ & \\
\hline \multicolumn{5}{|c|}{ WDD2 } \\
\hline SNIa & $0.38 \pm 0.08$ & \multirow{3}{*}{$4.9 / 5$} & $0.50 \pm 0.10$ & \multirow{3}{*}{$4.1 / 5$} \\
\hline SNII & $0.62 \pm 0.15$ & & $0.5 \pm 0.2$ & \\
\hline PopIII & $(-1.9 \pm 1.2) \times 10^{-3}$ & & $(1.9 \pm 1.2) \times 10^{-2}$ & \\
\hline
\end{tabular}

bigger values are also too large to claim a significant PopIII-star contribution.

In Fig. 9 we plot the abundances from the wdem model with the best-fit model (WDD2) listed in Table 8. Note that the WDD2 model is also the type Ia model favoured by Iwamoto et al. (1999) on observational grounds. The data points we use are taken from the third column of Table 4 . From this plot we see that the calcium abundance might be underestimated by the nucleosynthesis models and the cause of the high $\chi^{2}$ values. But the actual uncertainties in the measured calcium abundance might be bigger than the statistical error indicated here. This calcium overabundance is also seen in the analysis of 2A 0335+096 (Werner et al. 2006), confirming that the overabundance is probably not a statistical deviation. Every other abundance is consistent with the model within the error bars. The contribution of PopIII can be seen mainly in oxygen and neon, but the uncertainties are large.

We applied the same procedure to the abundances obtained from RGS. The relative abundances determined from RGS are very well constrained, but the number of elements that we can measure is too small to get a reasonable fit. If we fit the abundances we obtain errors which are equal to the measured values. Therefore, we do not show these fits in this paper.

\subsection{Non-thermal $X$-ray emission?}

In previous papers about Sérsic 159-03 (e.g. Kaastra et al. 2003; Bonamente et al. 2005) the larger $\chi_{r}^{2}$ for the single-temperature model is attributed to a soft X-ray excess. There are, however, a number of solutions to fit this soft excess and to obtain an acceptable $\chi_{r}^{2}$ of about 1.0. From the spectral fits in Sect. 4.1 we confirm that a single-temperature model is not the best description for the observed spectra. If we fit the data with a multitemperature model, then we obtain a $\chi_{r}^{2}$ of about 1.0. In this 

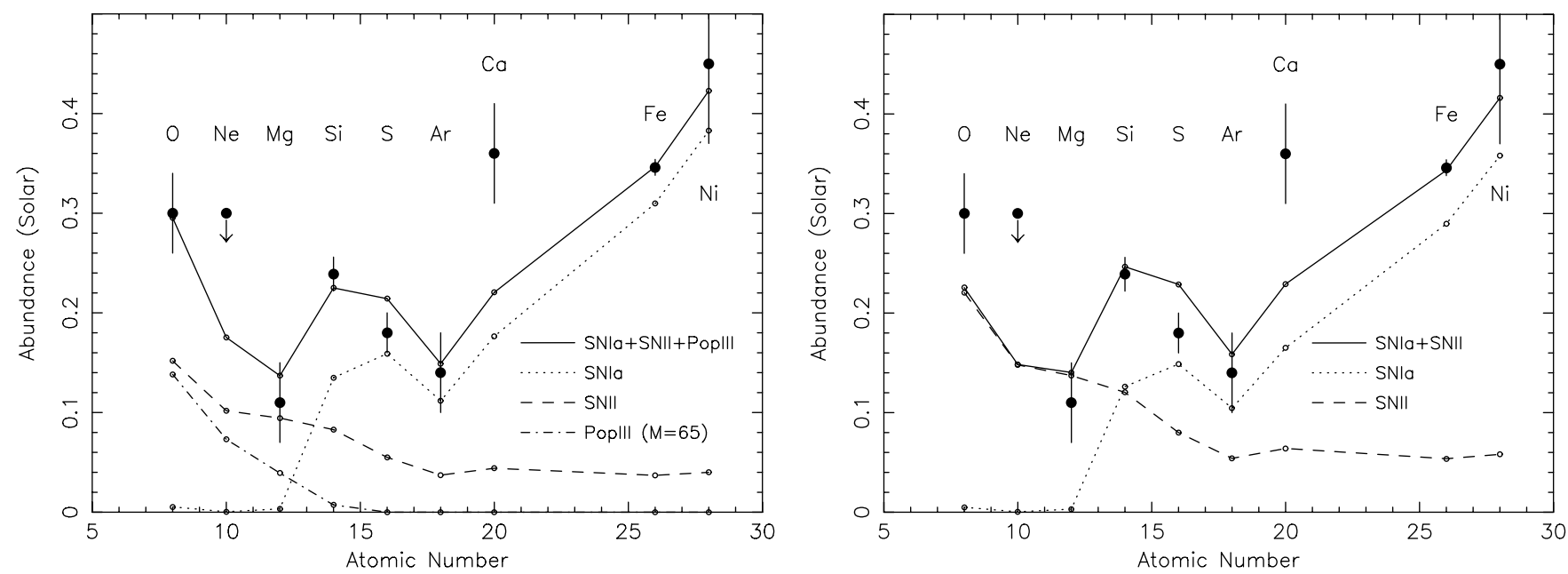

Fig. 9. The elemental abundances derived from a wdem fit, fitted with the WDD2 SNIa-yield model. The best fit from Table 8 is shown in the left panel with a PopIII mass of $65 M_{\odot}$. In the plot we also show the SNIa, SNII and PopIII contribution separately. In the right panel we show a fit without a PopIII contribution. In both plots the calcium abundance is included in the fit.

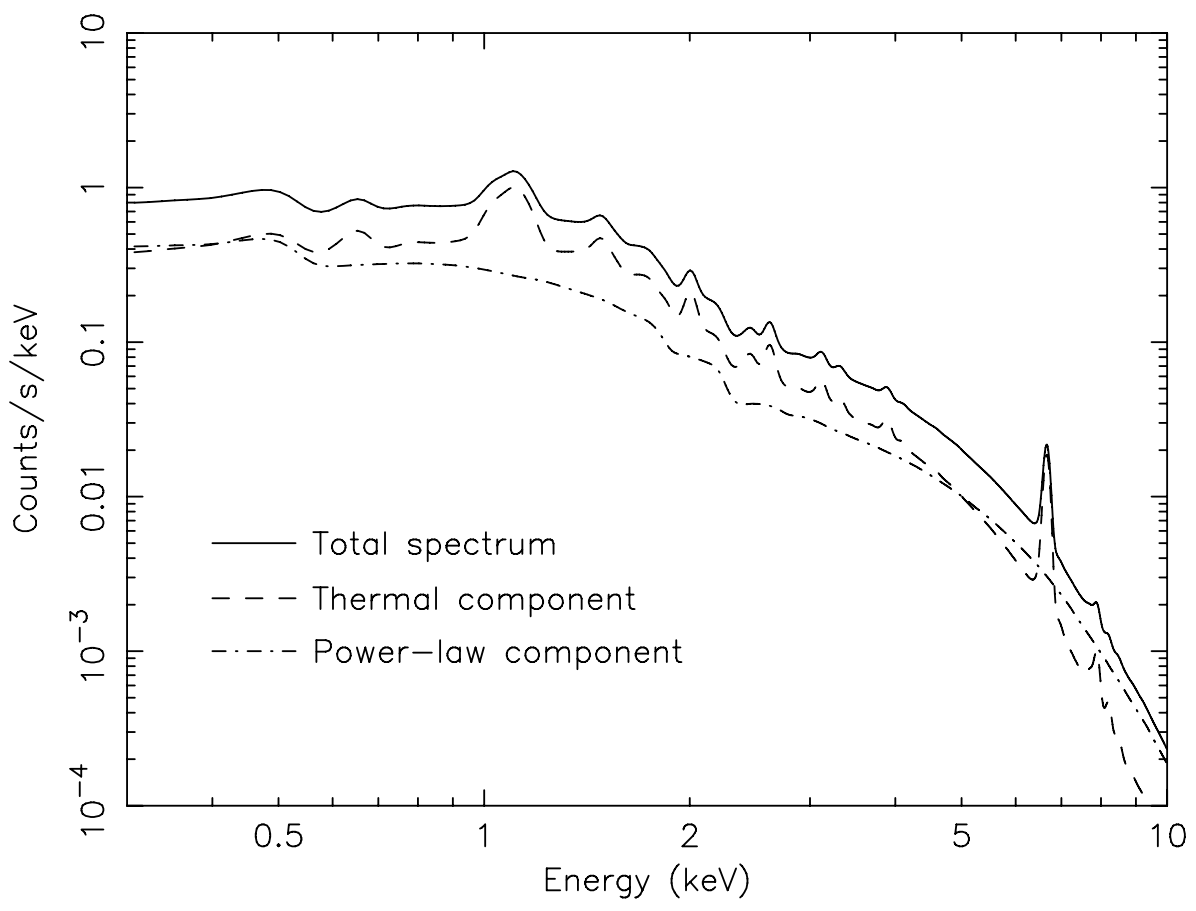

Fig. 10. This spectrum shows that an underlying power-law component can cause both a soft and a hard excess. The figure shows a power-law component with $\Gamma=2$, a singletemperature CIE component with a temperature of $2.5 \mathrm{keV}$ and the total spectrum. The contribution of the power-law component is mostly noticeable below $\sim 0.5 \mathrm{keV}$ and above $\sim 5 \mathrm{keV}$, hence causing a soft and hard excess with respect to the thermal component. section we explore the possibility of the existence of a soft excess in Sérsic 159-03 and speculate about its nature. The wdem and gdem models already provide acceptable fits to the spectra. We verified that the non-thermal component presented in this section does not affect the trends observed in the thermal analysis listed in Sect. 4.1.

One possibility is that the soft X-ray emission originates from warm thermal emission from the WHIM (Kaastra et al. 2003). Here we explore the possibility that inverse-Compton emission from CMB photons which are up-scattered to X-ray energies by relativistic electrons can also cause a soft excess. The same emission mechanism is also thought to be the origin of the hard excess in clusters of galaxies detected by BeppoSAX (e.g. Fusco-Femiano et al. 2005). A power-law component describing this inverse-Compton emission might be able to fit both this hard and soft excess.
A single non-thermal component as illustrated in Fig. 10 with a $\Gamma$ of 2.0 can explain both hard and soft X-ray excess with respect to a single-temperature model. At low energies $\lesssim 0.5 \mathrm{keV}$ the power-law component is comparable in flux to the thermal component, hence causing a soft excess. Above $\sim 5 \mathrm{keV}$ the power-law component in this example is even stronger than the thermal component, causing a hard X-ray excess. In Sérsic 159-03 the power-law emission is not as strong as in this example, but a similar model is consistent with the data.

In order to learn whether the data allow the soft excess to be thermal or non-thermal in nature, we fit the spectra from all annuli (see Table 3) with a single-temperature cluster component, a cold thermal component representing the WHIM, and a powerlaw component representing the soft and hard non-thermal emission. We first fit all annuli with the photon index and the $k T$ of the cool component as a free parameter. Because the photon index and temperatures were not well constrained in the outer 

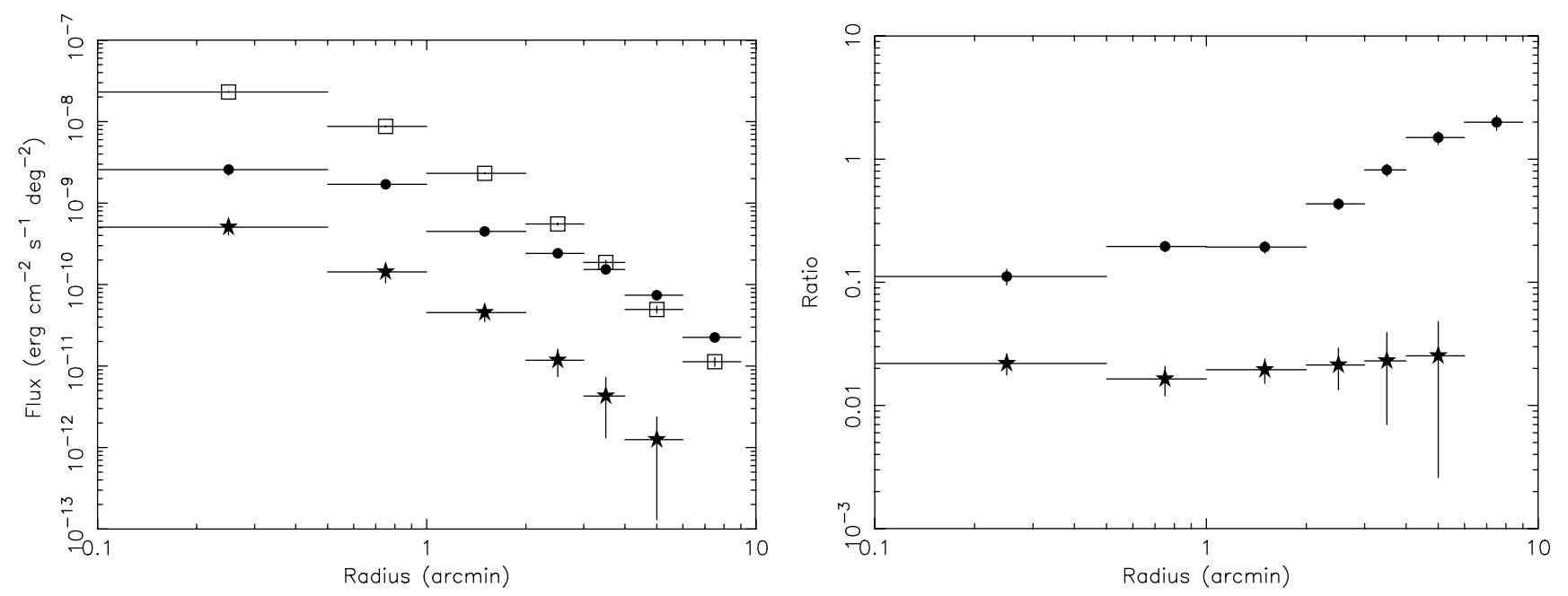

Fig. 11. Left: the $0.3-10 \mathrm{keV}$ integrated intensities for three model components: cluster hot gas ( $\square)$, power law $(\bullet)$ and soft-excess $(\star)$. Right: ratio between the intensities: power law/hot cluster gas $(\bullet)$ and soft-excess/hot cluster gas $(\star)$.

annuli, we fix the photon index and cool temperature in the final fits of all annuli to the mean value in the core region, which is 1.9 for the power-law index and $0.25 \mathrm{keV}$ for the cool thermal component. This assumption for $\Gamma$ is valid if the relativistic electrons are produced by acceleration in shocks, which behave similarly regardless of the position in the cluster. The radial profiles of the fitted components are shown in Fig. 11. The profile of the hot gas and the soft component have the same shape, which suggests that they have the same origin. The power-law component, however, has a more extended radial distribution. The flux is still the highest in the core of the cluster, but the slope of the radial profile is more shallow than the thermal emission. The ratio between the components is plotted in the right panel of Fig. 11. This figure indeed shows that the ratio between the soft excess and the cluster gas is constant. This is is not what you expect if the excess is due to WHIM which should be picked up preferably at the outskirts of the cluster. On the other hand, the power-law component does become relatively more important in the outskirts of the cluster. This is to be expected for Inverse-Compton emission because it scales with density $n$ instead of $n^{2}$ which is true for thermal emission. The different profile of the power-law emission therefore strongly suggests that if there is an excess, it is non-thermal in nature.

In the following fits we drop the cool component and fit the spectra with just a thermal (DEM) components and an additional power-law component to fit the soft-excess (Table 9). The models including a power law generally result in a lower $\chi^{2}$. This effect is most strongly present in the single-temperature fit: $\chi^{2}=897 / 914$ with a power-law component and $\chi^{2}=1228 / 916$ without. But also for the DEM models there is an improvement in $\chi^{2}$ that provides an argument for the presence of a non-thermal component.

The absolute values of the abundances change when an additional power law is used, but the general trends do not change considerably. The spatial abundance distributions are also consistent with the trends observed in the single-temperature models. In particular, fits including a power-law component show that the jump in $\mathrm{O} / \mathrm{Fe}$ in the centre of the cluster remains. However, the significance of the jump decreases compared to the single-temperature result when a power-law component is added.

Our suggestion that a single power-law component can both explain a possible soft as well as a hard excess of Sérsic 159-03,
Table 9. Fit results for an EPIC spectrum extracted from a circle with a radius of $4^{\prime}$ and centred on the core including a single-temperature thermal component + power law and multi-temperature thermal components + power law. Fluxes are calculated over the $0.3-10 \mathrm{keV}$ range and presented in $10^{-10} \mathrm{erg} \mathrm{cm}^{-2} \mathrm{~s}^{-1} \mathrm{deg}^{-2}$. Emission measure $\left(Y_{\text {thermal }}=\right.$ $\left.\int n_{\mathrm{e}} n_{\mathrm{H}} \mathrm{d} V\right)$ is given in $10^{66} \mathrm{~cm}^{-3}$ and $Y_{\text {pow }}$ is given in $10^{51} \mathrm{ph} \mathrm{s}^{-1} \mathrm{keV}^{-1}$ at $1 \mathrm{keV}$.

\begin{tabular}{lccc}
\hline \hline Parameter & single-temp + pow & wdem + pow & gdem + pow \\
\hline$Y_{\text {thermal }}$ & $16.8 \pm 0.3$ & $17.6 \pm 0.3$ & $18.9 \pm 0.4$ \\
$F_{\text {thermal }}$ & $16.2 \pm 0.2$ & $16.9 \pm 0.3$ & $18.0 \pm 0.5$ \\
$k T$ & $2.45 \pm 0.02$ & & $2.50 \pm 0.03$ \\
$k T_{\text {mean }}$ & & $2.53 \pm 0.07$ & \\
$k T_{\text {max }}$ & & $3.14 \pm 0.06$ & \\
$\alpha$ & & $0.32 \pm 0.04$ & \\
$\sigma_{T}$ & & & $0.208 \pm 0.012$ \\
$\mathrm{O}$ & $0.27 \pm 0.05$ & $0.26 \pm 0.05$ & $0.17 \pm 0.03$ \\
$\mathrm{Ne}$ & $1.00 \pm 0.11$ & $0.42 \pm 0.11$ & $0.11 \pm 0.09$ \\
$\mathrm{Mg}$ & $0.18 \pm 0.04$ & $0.21 \pm 0.05$ & $0.18 \pm 0.04$ \\
$\mathrm{Si}$ & $0.32 \pm 0.02$ & $0.33 \pm 0.02$ & $0.33 \pm 0.02$ \\
$\mathrm{~S}$ & $0.22 \pm 0.02$ & $0.25 \pm 0.02$ & $0.25 \pm 0.02$ \\
$\mathrm{Ar}$ & $0.20 \pm 0.05$ & $0.23 \pm 0.05$ & $0.26 \pm 0.06$ \\
$\mathrm{Ca}$ & $0.42 \pm 0.06$ & $0.45 \pm 0.06$ & $0.52 \pm 0.07$ \\
$\mathrm{Fe}$ & $0.433 \pm 0.011$ & $0.409 \pm 0.011$ & $0.275 \pm 0.009$ \\
$\mathrm{Ni}$ & $0.62 \pm 0.11$ & $0.60 \pm 0.10$ & $0.54 \pm 0.10$ \\
$Y_{\text {pow }}$ & $6.6 \pm 0.4$ & $5.3 \pm 0.4$ & $3.5 \pm 0.8$ \\
$F_{\text {pow }}$ & $3.5 \pm 0.2$ & $2.8 \pm 0.2$ & $1.6 \pm 0.4$ \\
$\Gamma$ & $2.06 \pm 0.03$ & $2.10 \pm 0.04$ & $2.30 \pm 0.15$ \\
$\chi^{2} /$ d.o.f. & $897 / 914$ & $853 / 913$ & $861 / 913$ \\
\hline
\end{tabular}

may also be relevant for several clusters for which non-thermal emission has been detected by BeppoSAX (Fusco-Femiano et al. 2000, 2004, 2005). However, the Coma-cluster is, so-far, the only cluster for which both a hard X-ray component and a soft excess has been reported (Fusco-Femiano et al. 2004; Kaastra et al. 2003). For Sérsic 159-03, it does indeed seem to be the case that a single non-thermal component explains both the soft and hard X-ray excess: Extrapolating the BeppoSAX flux in the $20-80 \mathrm{keV}$ range, $(1.5 \pm 0.5) \times 10^{-11} \mathrm{erg} \mathrm{s}^{-1} \mathrm{~cm}^{-2}$, to the $0.3-10 \mathrm{keV}$ band, assuming $\Gamma=2.0$, we obtain a flux of $(3.8 \pm 1.3) \times 10^{-11} \mathrm{erg} \mathrm{s}^{-1} \mathrm{~cm}^{-2}$. This is consistent with the XMM-Newton flux for the putative non-thermal component 
reported by Kaastra et al. $(2003),(6.8 \pm 2.0) \times 10^{-11} \mathrm{erg} \mathrm{s}^{-1} \mathrm{~cm}^{-2}$. For this calculation we assume that all the non-thermal emission originates from within a circle of $12^{\prime}$ from the core.

\section{Discussion}

\subsection{Temperature structure}

The parameters of the DEM distributions which we obtain from multi-temperature fitting, are well determined for this cluster. However, we cannot discriminate between different shapes of DEM distributions and the presence of a non-thermal component. The DEM parameters $\alpha($ wdem $)$ and $\sigma_{\mathrm{T}}(\mathrm{gdem})$ values show a slight increase to the outer parts of the cluster. This can be explained by the steep temperature gradients we see both in the cooling core and in the outer parts of the cluster. The values we derive for $\alpha$ are higher than the value of $0.20 \pm 0.05$ derived by Kaastra et al. (2004) based on a shorter exposure. However, their background subtraction method and handling of spectral excess is different, which can lead to systematic differences. Also projection effects can have an influence on the broadness of the DEM distribution, but as Kaastra et al. (2004) and Werner et al. (2006) show, multi-temperature models are also needed for fitting deprojected spectra.

\subsection{Abundance distribution and enrichment by supernova types la/II and Population-III stars}

From the EPIC and RGS spectra we obtain radial abundance profiles for the most abundant metals. The EPIC and RGS radial profiles for oxygen show a jump in the centre of the cluster. The key data point is the point in the $0-0.5^{\prime}$ bin. If we compare the $\mathrm{O} / \mathrm{Fe}$ ratio of the central bin with the neighbouring bins, its value is significantly lower. This means that either the iron abundance is relatively high or oxygen is low in the centre of the cluster.

Recently, using cluster simulations, Schindler et al. (2005) found that ram-pressure stripping acting on in-falling galaxies can result in centrally peaked abundance profiles, while early galactic winds produce an extended distribution of the oxygen abundance. The spatial distribution of oxygen, which is difficult to determine, is the key to understand the evolution history of the cluster.

Contrary to the iron and oxygen lines in Sérsic 159-03, the widths of these lines are the same in the cluster 2A 0335+096 (Werner et al. 2006). A possible explanation is that the cluster 2A 0335+096 shows a much stronger temperature drop in the centre of the cluster. Since spectral lines are stronger when the temperature is lower, the RGS spectrum is dominated by line emission from the cool core of the cluster. In this case, the line profiles of iron and oxygen should follow the temperature structure more than the abundance distribution of the elements. In Sérsic 159-03 the temperature profile within the RGS extraction region is relatively flat. The temperature is about $2.5 \mathrm{keV}$ with a spread of $10 \%$. Because a strong thermal gradient is absent in the core of this cluster, the line profile should follow the abundance distribution of the elements. The observed line profiles in RGS therefore strongly suggest that in Sérsic 159-03 oxygen emission is lower in the centre of the line profile compared to iron, which is consistent with the radial profiles extracted from EPIC and RGS.

From several abundance yield simulations for Supernova type Ia and II we know that oxygen dominates the yield of type II supernovae, while iron originates mostly from type Ia supernovae. If the iron abundance is more peaked in the centre of the cluster, we can formulate a scenario of the possible enrichment history of the ICM in Sérsic 159-03. In the early universe the Inter-Galactic Medium (IGM) might have been enriched by PopIII-star explosions. The star bursts in the young galaxies that form after PopIII enrichment produce a lot of SNII which produce mainly oxygen. Because clusters are still in an early stage of development, the oxygen becomes well mixed through the IGM. Then, 1 billion years after the "Big Bang", also SNIa explosions start enriching the local ISM in galaxies continuously. If a galaxy containing both SNIa and SNII products falls into a cluster, the ISM is stripped off by ram-pressure stripping, preferably in the denser core of the cluster, hence leading to a more centrally peaked abundance distribution of all SNIa and SNII products (Schindler et al. 2005). Because iron is also produced by SNIa explosions in the central cD galaxy, this could enhance the iron abundance in the centre of the cluster (Tamura et al. 2001). The drop in the centre of the observed $\mathrm{O} / \mathrm{Fe}$ fits within this scenario.

In order to check whether the absolute abundance of iron can be consistent with the interpretation we just formulated, we calculate the total iron mass in the cluster. We find about $4 \times 10^{9} M_{\odot}$ within a radius of $300 \mathrm{kpc}$ of the core. If all this iron originates from SNIa explosions, we would need at least $5 \times 10^{9}$ explosions in the cluster lifetime, which is of the order of $10^{10}$ years. Since there are typically $10^{2}$ galaxies, there should be about 1 SNIa explosion per 200 years per galaxy, which is consistent with the rate of SNIa in our own galaxy.

We then fit the abundances from EPIC to a model of the yields of SNIa, SNII and PopIII stars (Iwamoto et al. 1999; Heger \& Woosley 2002). Using a linear combination of the yields we can estimate the expected abundances and fit them to the data. From the fit we obtain the relative contributions of the three types of metal-enriching sources.

We have to be careful, though, when we interpret the fitted supernova ratios. The mechanisms by which the elements are ejected into the ICM are not yet fully understood and are certainly not included in our simplified model. A small part of the supernova products can also be locked up in low-mass stars, so the abundance distribution we observe in the ICM is not entirely representative for the total abundance distribution in the cluster. A more detailed description of the problems when one uses this simplified model is described in Matteucci \& Chiappini (2005). The derived supernova ratios should be interpreted as the number fraction of supernovae that would be needed to enrich the ICM, not the actual number of supernovae during the history of the cluster.

Our analysis shows that PopIII stars are not required to fit the data. The contributions from this early generation of stars are probably too small to be detectable in current cluster abundance patterns. We cannot confirm the results from a sample of clusters observed with ASCA that suggest that low mass PopIII stars are necessary to explain the observed silicon and sulfur abundance (Baumgartner et al. 2005). Our analysis, which includes more elements with about the same error bars on the abundances as in Baumgartner et al. (2005), shows that a PopIIIstar contribution is not detected in Sérsic 159-03. Considering the single-temperature spectral models used by Baumgartner et al. (2005), we think that a temperature bias in the iron abundance makes their $\mathrm{Si} / \mathrm{Fe}$ and $\mathrm{S} / \mathrm{Fe}$ ratios unreliable. We also cannot confirm the underabundance of argon and calcium in the Baumgartner et al. (2005) sample. We actually measure an overabundance of calcium with respect to the nucleosynthesis models, which is in line with the result by Werner et al. (2006) in the 
cluster 2A 0335+096. This might be an indication that the SNII and SNIa models have difficulties predicting the right yield for this element, but the actual uncertainties in our measured value might also be bigger than the quoted statistical error. When we compare the supernova fractions found by Werner et al. (2006) in 2A 0335+096, we see that the SNIa contribution in Sérsic 159-03 tends to be 5-25\% higher.

\subsection{Soft excess and non-thermal $X$-ray emission}

An alternative solution to obtain an acceptable fit for the spectra of Sérsic 159-03 is to add a soft-excess component to a singletemperature model. We explore the possibility that the deviations from the single-temperature model are due to a different component, like the WHIM or inverse-Compton emission.

A non-thermal component can explain both a soft and hard excesses for both Sérsic 159-03 and Coma, is consistent with inverse-Compton emission of CMB photons on relativistic electrons (Hwang 1997). The alternative explanation, nonthermal bremsstrahlung emission from a non-thermal tail to the Maxwellian energy distribution of the electrons, would only result in a hard X-ray excess.

There are several possible sources that can produce a population of relativistic electrons in a cluster that would explain the observed power-law emission. The acceleration of electrons can occur, for example, in active galactic nuclei, supernovae and pulsars (Sarazin \& Lieu 1998). But also plasma waves or shocks in the ICM can accelerate electrons (see Enßlin et al. 1999, for a discussion). Using our results from Sérsic 159-03, we propose a new scenario where electrons are accelerated by the shock waves associated with in-falling galaxies.

We already discussed the possibility that the centrally peaked iron distribution might be due to an efficient ram-pressure stripping of in-falling galaxies in the dense core of the cluster. Some fraction of these galaxies being stripped would have moderate supersonic velocities. The galaxies will drive bow shocks of Mach numbers up to $\sim 3$ in the intra-cluster gas that can accelerate electrons to relativistic energies. To produce photons of $\sim 10 \mathrm{keV}$ due to inverse-Compton up-scattering of CMB photons, electrons with a Lorentz factor $\gamma \sim 7 \times 10^{3}$ are required. The life-time of such an electron in the cluster core is about $2.3 \times 10^{12} \gamma^{-1}$ years (assuming that the magnetic field in the core is below $2 \mu \mathrm{G}$ and the energy densities of optical and IR radiation field in the core are below $0.1 \mathrm{eV} \mathrm{cm}^{-3}$ ). It is worth noting here that the central galaxy in Sérsic 159-03 is one of the brightest cD galaxies detected in IR (Hansen et al. 2000), but the energy density of the IR photon field produced by the galaxy is below $0.1 \mathrm{eV} \mathrm{cm}^{-3}$.

The electron acceleration time at a shock of velocity $\geq 1000 \mathrm{~km} \mathrm{~s}^{-1}$ in the cluster core is typically much shorter than $3 \times 10^{8}$ years. Thus, to provide a steady non-thermal X-ray emission the stripping galaxies must cross the cluster core at a frequency $v$ of about 1 in $3 \times 10^{8}$ years. The mechanical power, $L_{\text {kin }}$, dissipated by the galactic shocks of velocity $v_{\mathrm{g}}$ and average radius $R_{\mathrm{g}}$ in the core region of diameter $D_{\mathrm{c}}$ and average number density $n_{\mathrm{c}}$ can be written as:

$$
\begin{aligned}
L_{\mathrm{kin}} \approx & 2 \times 10^{43}\left(\frac{R_{\mathrm{g}}}{15 \mathrm{kpc}}\right)^{2}\left(\frac{D_{\mathrm{c}}}{300 \mathrm{kpc}}\right)\left(\frac{v_{\mathrm{g}}}{10^{3} \mathrm{~km} \mathrm{~s}^{-1}}\right)^{2} \\
& \times\left(\frac{n_{\mathrm{c}}}{3 \times 10^{-3} \mathrm{~cm}^{-3}}\right)\left(\frac{v}{3 \times 10^{-9} \mathrm{yr}^{-1}}\right) \mathrm{erg} \mathrm{s}^{-1} .
\end{aligned}
$$

For the parameters assumed above, galaxies with velocity $v_{\mathrm{g}} \sim$ $2000 \mathrm{~km} \mathrm{~s}^{-1}$ would provide $L_{\mathrm{kin}} \sim 10^{44} \mathrm{erg} \mathrm{s}^{-1}$. A few percent of the power would be enough to maintain the relativistic electrons required to provide the $10 \mathrm{keV}$ regime non-thermal emission. Note that the lifetime of the electrons with Lorentz factor below 100 would be about the Hubble time. This implies that the spectrum of electrons accelerated by multiple successive shocks of Mach number about 3 will have power-law distribution of index about 3 at $\gamma \sim 10^{4}$, and a flat distribution at $\gamma \leq 100$, where the Compton losses are not important. The electron distribution index 3 corresponds to the photon index of the photon emission of 2 , close to that observed.

\section{Conclusions}

We have analysed high-resolution X-ray spectra of the cluster of galaxies Sérsic 159-03 obtained with XMM-Newton and conclude that:

1. For the first time we accurately determine the abundances in a cluster of galaxies which fit to a linear combination of current supernova yield models.

2. From the line width in RGS and the radial profiles from EPIC/RGS we find a jump in the $\mathrm{O} / \mathrm{Fe}$ ratio around a radius of $0.5^{\prime}$ from the cluster centre. The $\mathrm{O} / \mathrm{Fe}$ ratio in the centre of the cluster is lower compared to its immediate surroundings. A combination of ram-pressure stripping (Schindler et al. 2005) and enrichment by SNIa in the central elliptical galaxy (Tamura et al. 2001) can explain the observed profile.

3. We do not detect a contribution of PopIII stars in Sérsic 159-03. This result is not in line with the claim by Baumgartner et al. (2005) that PopIII stars are necessary to explain the abundances measured in an ASCA sample of clusters. The number contribution of SNIa with respect to the total number of supernovae we find, based on the measured abundances, is about $25-50 \%$.

4. The spectra can also be fitted with an additional non-thermal component with a power-law index of about 2.1 on top of the thermal emission. The $\chi_{r}^{2}$ of these fits is comparable to the $\chi_{r}^{2}$ using only multi-thermal models. If a non-thermal component is present in Sérsic 159-03, it can be explained by Inverse-Compton scattering of $\mathrm{CMB}$ photons and relativistic electrons.

Acknowledgements. We would like to thank the anonymous referee for providing comments that helped to improve the presentation of our results and conclusions in this paper. This work is based on observations obtained with XMMNewton, an ESA science mission with instruments and contributions directly funded by ESA member states and the USA (NASA). The Netherlands Institute for Space Research (SRON) is supported financially by NWO, the Netherlands Organisation for Scientific Research.

\section{References}

Allen, S. W., \& Fabian, A. C. 1997, MNRAS, 286, 583

Baumgartner, W. H., Loewenstein, M., Horner, D. J., \& Mushotzky, R. F. 2005, ApJ, 620, 680

Bonamente, M., Lieu, R., Mittaz, J. P. D., Kaastra, J. S., \& Nevalainen, J. 2005, ApJ, 629, 192

Cen, R., \& Ostriker, J. P. 1999, ApJ, 514, 1

Davé, R., Cen, R., Ostriker, J. P., et al. 2001, ApJ, 552, 473

Davis, J. E. 2001, ApJ, 548, 1010

De Luca, A., \& Molendi, S. 2004, A\&A, 419, 837

de Plaa, J., Kaastra, J. S., Tamura, T., et al. 2004, A\&A, 423, 49

de Plaa, J., Kaastra, J. S., Méndez, M., et al. 2005, AdSpR, 36, 601

De Young, D. S. 1978, ApJ, 223, 47

den Herder, J. W., Brinkman, A. C., Kahn, S. M., et al. 2001, A\&A, 365, L7

Dickey, J. M., \& Lockman, F. J. 1990, ARA\&A, 28, 215

Edge, A. C., \& Stewart, G. C. 1991, MNRAS, 252, 414

Enßlin, T. A., Lieu, R., \& Biermann, P. L. 1999, A\&A, 344, 409 
Finoguenov, A., Briel, U. G., \& Henry, J. P. 2003, A\&A, 410, 777

Fusco-Femiano, R., dal Fiume, D., Feretti, L., et al. 1999, ApJ, 513, L21

Fusco-Femiano, R., Dal Fiume, D., De Grandi, S., et al. 2000, ApJ, 534, L7

Fusco-Femiano, R., Orlandini, M., Brunetti, G., et al. 2004, ApJ, 602, L73

Fusco-Femiano, R., Landi, R., \& Orlandini, M. 2005, ApJ, 624, L69

Gibson, B. K., Loewenstein, M., \& Mushotzky, R. F. 1997, MNRAS, 290, 623

González-Riestra, R. 2004,

http://xmm . vilspa.esa.es/docs/documents/CAL-TN-0058-1-0.ps.gz

Hansen, L., Jørgensen, H. E., Nørgaard-Nielsen, H. U., et al. 2000, A\&A, 356, 83

Heger, A., \& Woosley, S. E. 2002, ApJ, 567, 532

Hwang, C. 1997, Science, 278, 1917

Iwamoto, K., Brachwitz, F., Nomoto, K., et al. 1999, ApJS, 125, 439

Jansen, F., Lumb, D., Altieri, B., et al. 2001, A\&A, 365, L1

Kaastra, J. S., Mewe, R., \& Nieuwenhuijzen, H. 1996, in UV and X-ray Spectroscopy of Astrophysical and Laboratory Plasmas: Proceedings of the Eleventh Colloquium on UV and X-ray ... held on May 29-June 2, 1995, Nagoya, Japan, ed. K. Yamashita, \& T. Watanabe (Tokyo: Universal Academy Press) (Frontiers science series; No. 15), 411

Kaastra, J. S., Ferrigno, C., Tamura, T., et al. 2001, A\&A, 365, L99

Kaastra, J. S., Lieu, R., Tamura, T., Paerels, F. B. S., \& den Herder, J. W. 2003, A\&A, 397, 445

Kaastra, J. S., Tamura, T., Peterson, J. R., et al. 2004, A\&A, 413, 415

Katayama, H., Takahashi, I., Ikebe, Y., Matsushita, K., \& Freyberg, M. J. 2004, A\&A, 414, 767

Kuntz, K. D., \& Snowden, S. L. 2000, ApJ, 543, 195
Lieu, R., Mittaz, J. P. D., Bowyer, S., et al. 1996, ApJ, 458, L5

Lodders, K. 2003, ApJ, 591, 1220

Loewenstein, M. 2001, ApJ, 557, 573

Lumb, D. H., Warwick, R. S., Page, M., \& De Luca, A. 2002, A\&A, 389, 93

Maia, M. A. G., da Costa, L. N., Willmer, C., Pellegrini, P. S., \& Rite, C. 1987, AJ, 93, 546

Matteucci, F., \& Chiappini, C. 2005, Pub. Astron. Soc. Australia, 22, 49

Mittaz, J. P. D., Lieu, R., \& Lockman, F. J. 1998, ApJ, 498, L17

Peterson, J. R., Paerels, F. B. S., Kaastra, J. S., et al. 2001, A\&A, 365, L104

Peterson, J. R., Kahn, S. M., Paerels, F. B. S., et al. 2003, ApJ, 590, 207

Pratt, G. W., \& Arnaud, M. 2002, A\&A, 394, 375

Raymond, J. C., \& Smith, B. W. 1977, ApJS, 35, 419

Read, A. M., \& Ponman, T. J. 2003, A\&A, 409, 395

Rossetti, M., \& Molendi, S. 2004, A\&A, 414, L41

Sérsic, J. L. 1974, Ap\&SS, 28, 365

Sarazin, C. L., \& Lieu, R. 1998, ApJ, 494, L177

Schindler, S., Kapferer, W., Domainko, W., et al. 2005, A\&A, 435, L25

Tamura, T., Bleeker, J. A. M., Kaastra, J. S., Ferrigno, C., \& Molendi, S. 2001, A\&A, 379, 107

Tamura, T., Kaastra, J. S., den Herder, J. W. A., Bleeker, J. A. M., \& Peterson, J. R. 2004, A\&A, 420, 135

Tsujimoto, T., Nomoto, K., Yoshii, Y., et al. 1995, MNRAS, 277, 945

Turner, M. J. L., Abbey, A., Arnaud, M., et al. 2001, A\&A, 365, L27

Verner, D. A., Ferland, G. J., Korista, K. T., \& Yakovlev, D. G. 1996, ApJ, 465, 487

Werner, N., de Plaa, J., Kaastra, J. et al. 2006, A\&A, 449, 475 